\title{
Trial-by-trial coupling between EEG and BOLD identifies networks related to alpha and theta EEG power increases during working memory maintenance
}

\author{
René Scheeringa ${ }^{a, *}$, Karl Magnus Petersson ${ }^{\mathrm{a}, \mathrm{b}}$, Robert Oostenveld ${ }^{\mathrm{a}}$, David G. Norris ${ }^{\text {a }}$, \\ Peter Hagoort $^{\mathrm{a}, \mathrm{b}}$, Marcel C.M. Bastiaansen ${ }^{\mathrm{a}, \mathrm{b}}$ \\ a Radboud University Nijmegen, Donders Institute for Brain, Cognition and Behaviour, Centre for Cognitive Neuroimaging, Trigon building, PO Box 9101, 6500 HB Nijmegen, The Netherlands \\ ${ }^{\mathrm{b}}$ Max Planck Institute for Psycholinguistics, Nijmegen, The Netherlands
}

\section{A R T I C L E I N F O}

\section{Article history:}

Received 25 March 2008

Revised 7 July 2008

Accepted 21 August 2008

Available online 18 September 2008

\begin{abstract}
A B S T R A C T
PET and fMRI experiments have previously shown that several brain regions in the frontal and parietal lobe are involved in working memory maintenance. MEG and EEG experiments have shown parametric increases with load for oscillatory activity in posterior alpha and frontal theta power. In the current study we investigated whether the areas found with fMRI can be associated with these alpha and theta effects by measuring simultaneous EEG and fMRI during a modified Sternberg task This allowed us to correlate EEG at the single trial level with the fMRI BOLD signal by forming a regressor based on single trial alpha and theta power estimates. We observed a right posterior, parametric alpha power increase, which was functionally related to decreases in BOLD in the primary visual cortex and in the posterior part of the right middle temporal gyrus. We relate this finding to the inhibition of neuronal activity that may interfere with WM maintenance. An observed parametric increase in frontal theta power was correlated to a decrease in BOLD in regions that together form the default mode network. We did not observe correlations between oscillatory EEG phenomena and BOLD in the traditional WM areas. In conclusion, the study shows that simultaneous EEG-fMRI recordings can be successfully used to identify the emergence of functional networks in the brain during the execution of a cognitive task.
\end{abstract}

(ㄷ) 2008 Elsevier Inc. All rights reserved.

\section{Introduction}

Working memory (WM) has been one of the central themes in cognitive neuroscience research for the past decades. A considerable number of studies have either used hemodynamic (e.g. PET and fMRI) or electrophysiological recordings (e.g. MEG, EEG, intracranial recordings). PET and fMRI have been successful in linking different brain regions to task and modality specific WM processes (Cabeza and Nyberg, 2000; D'Esposito et al., 2000; Fletcher and Henson, 2001). Across modalities and tasks, dorso- and ventrolateral prefrontal and posterior parietal regions have been often linked to WM processes (Cabeza and Nyberg, 2000). In recent years, regions in the medial temporal lobe have also been implicated in WM, suggesting WM and long term memory share in part the same neural substrate (Cabeza et al., 2002; Petersson et al., 2006; Piekema et al., 2006; Ranganath et al., 2005; Ranganath and D'Esposito, 2001).

The high temporal resolution of EEG and MEG makes it possible to study WM-related processes at a millisecond time-scale. WMrelated increases in oscillations observed in the EEG or MEG have

\footnotetext{
* Corresponding author. Fax: +31243610 989.

E-mail address: rene.scheeringa@donders.ru.nl (R. Scheeringa).
}

been reported in the theta band (Gevins et al., 1997; Krause et al., 2000; Onton et al., 2005), alpha band (Klimesch et al., 1999); Jensen et al., 2002; Jokisch and Jensen, 2007; Schack and Klimesch, 2002; Tuladhar et al., 2007) and gamma band (Jokisch and Jensen, 2007; Kaiser et al., 2003; Lutzenberger et al., 2002; Tallon-Baudry et al., 1998). More specifically, parametric increases with WM load in the maintenance interval have been reported for posterior alpha (Jensen et al., 2002) and frontal theta power (Gevins et al., 1997; Jensen and Tesche, 2002; Onton et al., 2005). Different functional roles have been proposed for these different frequency bands. Theta and gamma have been hypothesized to be a direct neural correlate of WM maintenance, possibly in cooperation with medial temporal structures (Jensen, 2006; Jensen and Lisman, 1998). Alpha power increases have been linked to active inhibition of neuronal activity that could otherwise disturb the WM process (Jokisch and Jensen, 2007; Klimesch et al., 2007). Differential alpha power effects can be seen in upper and lower sub-bands (Klimesch, 1999). Decreases in power in the lower alpha band have been linked to higher task demands and attentional processing, whereas decreases in power in the upper alpha band reflects have been related to increased declarative memory performance. Klimesch et al. (1999) observed an increase during WM maintenance of character strings and they suggest that this is related to inhibition of memory activities that could otherwise disturb the WM process. 
Based on these previous findings the question arises whether the regions observed with PET and fMRI are also functionally related to the oscillatory phenomena as measured with MEG and EEG. To address this question, we simultaneously measured EEG and fMRI while subjects performed a modified verbal Sternberg WM task. By relating single trial estimates of WM-related power increases to changes in the BOLD signal we hope to uncover which brain regions are functionally related to these WM-induced power increases. Successful single trial coupling between EEG measures (ERP's) and the BOLD signal has been demonstrated by Eichele et al. (2005) and Debener et al. (2005).

The relation between alpha power on the one hand and the BOLD signal on the other hand has been investigated in previous studies. Posterior alpha is most prominent during eyes closed alert wakefulness, and usually decreases with increased visual processing (Klimesch, 1999). We therefore expected posterior alpha to correlate with the BOLD signal regions involved in visual processing. Posterior alpha has indeed been found to correlate with posterior visual areas in eyes closed resting state conditions (Feige et al., 2005; Goldman et al., 2002; Moosmann et al., 2003). More recently Laufs et al. (2006) and Goncalves et al. (2006) showed that the observed networks that correlate with alpha power might depend on the relative strengths of other frequency bands in the entire power spectrum. Recently, Meltzer et al. (2007) showed in separate EEG and FMRI sessions, that load dependent increases in alpha power during WM maintenance correlate negatively with the midline parietal-occipital cortex across subjects.

Frontal theta is a prominent feature in the EEG that is reported to increase in tasks that require attention or WM. It is often studied during mental arithmetic (Inanaga, 1998; Inouye et al., 1994; Ishihara and Yoshii, 1972; Ishii et al., 1999; Lazarev, 1998; Mizuki et al., 1980; Sasaki et al., 1996; Smith et al., 1999), but more recently it has also been associated with WM maintenance (Gevins et al., 1997; Jensen, 2006; Jensen et al., 2002). Recently, two studies investigated the BOLD correlates of frontal theta activity during mental arithmetic (Mizuhara et al., 2004; Sammer et al., 2007). While Sammer et al. (2007) reported only positive correlations between BOLD and theta in the insula, medial temporal lobe, superior temporal cortex, cingulate cortex and various frontal regions. Mizuhara et al. (2004) reported predominantly negative correlations in medial frontal, posterior cingulate, temporal and inferior parietal regions. In a recent resting state experiment we only observed negative correlations with frontal theta power in a collection of regions that together form the default mode network (DMN; Scheeringa et al., 2008). This negative correlation between frontal theta and DMN activity was also observed by Meltzer et al. (2007) across subjects in a WM task.

Regions in which correlations between single trial EEG power measures and the BOLD signal are observed cannot be directly interpreted as being functionally related to the WM-induced parametric power increases. The reason for this is that the relation between BOLD signal in a region, and single trial estimates of power could also be related only to task independent fluctuations in power. These task independent power fluctuations could be related to EEG activity coming from other sources than the one that shows a WMinduced power change, that leak into the single trial estimates of power. Another potential source of task independent variation in power could lie in trial-by-trial coupling of task related regions with task unrelated regions, which has been observed between the motor cortices using fMRI (Fox et al., 2006). This task independent trial-bytrial coupling could possibly also be reflected in the trial-by-trial variation of EEG power components that do show an average effect of WM load. Therefore we argue here that regions functionally related to WM-induced alpha and theta EEG power increases should show a BOLD response that is in line with the observed WM effects in these bands and also show a relation with the single trial variation in power.

\section{Methods}

\section{Subjects}

Twenty right handed volunteers ( 16 female, 4 male, age range: 18 28) participated in the study. All subjects reported to be free of neurological or psychiatric impairment, experienced neurological trauma or from using neuroleptics. Subjects gave written informed consent prior to the measurements and all subjects were paid a small fee for their participation.

\section{Design and procedure}

Subjects performed a variant of the Sternberg WM task (see also Fig. 1). They had to remember a string of either $0,3,5$ or 7 consonants that was presented for $3 \mathrm{~s}$. When less than 7 consonants were presented, filler symbols (\# 's) were added on the left and right side of the string in order to make the sensory input for the four conditions comparable. After a $7 \mathrm{~s}$ maintenance interval a probe was presented for $300 \mathrm{~ms}$. All responses were made with the right hand. Subjects had to press a button with the index finger if the probe matched one of the consonants in the memory set, or with their middle finger if the probe did not match. When no consonants were presented during the encoding phase (i.e., the baseline condition), the probe consisted of either an $\mathrm{L}$ or an $\mathrm{R}$, requiring a right handed index or middle finger response respectively. We avoided the use of the letters $L$ and the $R$ in the other WM conditions. Subjects were instructed to make fast and accurate responses. The inter-trial interval varied between 7,8 and $9 \mathrm{~s}$. A fixation cross was presented during the maintenance interval and the ITI. During the trial, the letters and the fixation cross were presented in black on a gray background. After the button press the fixation cross turned green, indicating the subject was allowed to make eye blinks. One second before the start of the next trial the fixation cross turned black again. Subjects were instructed not to blink from this moment until they had pressed a button.

All subjects participated in two experimental sessions. For both sessions, subjects read a written instruction before and after the application of the EEG electrodes. The first session took place in the EEG laboratory, the second session a few weeks later in the MR scanner where simultaneously EEG and fMRI were acquired. Each session consisted of three blocks of 72 trials. Within each block, 18 trials of each WM condition were presented. Half of the trials in each condition required an index finger response (Match trials in Load 3, Load 5 and Load 7). For each WM load condition there were 18 trials in each block. One block took approximately $22 \mathrm{~min}$. In the session inside the MR scanner, a 10 min resting state measurement with simultaneous EEG and fMRI and a $10 \mathrm{~min}$ anatomical MR scan were recorded after the three WM blocks. Prior to the start of each session subjects did a short practice block of eight trials, two for each WM condition. When necessary, subjects were allowed to do a second block of eight practice trials. The recording session in the EEG

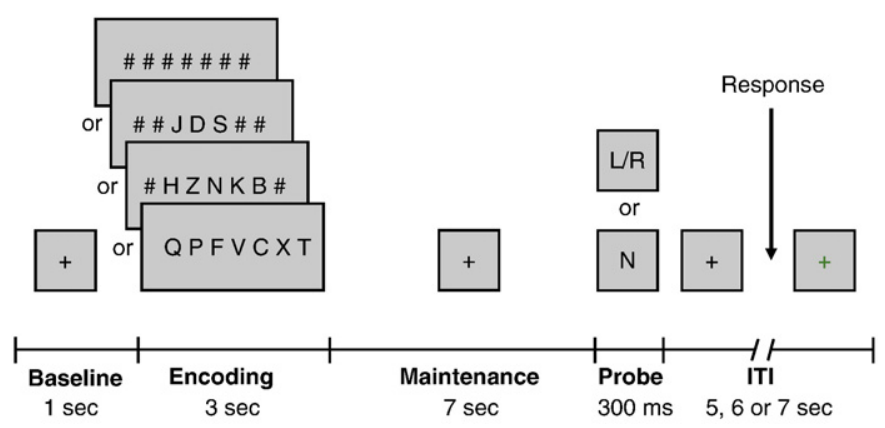

Fig. 1. Schematic representation of the modified Sternberg paradigm used in this study. The task is described in more details in the Methods section. 
laboratory took approximately $1 \mathrm{~h}$ and $10 \mathrm{~min}$ and the recording session in the MR scanner measurements took approximately $1 \mathrm{~h}$ and $30 \mathrm{~min}$.

\section{Behavioral data}

Repeated measures ANOVA's were carried out on the reaction times and on the numbers of errors. Factors were WM load (four levels, Load 0, Load 3, Load 5 and Load 7) and response (two levels, index finger/match and middle finger/mismatch). The main and interaction effects were tested. Where appropriate, the GreenhouseGeisser correction for non-sphericity was applied.

\section{Electrophysiological recordings}

EEG was recorded at 29 scalp sites (Fp1, Fp2, F3, F4, C3, C4, P3, P4, 01, O2, F7, F8, T7, T8, P7, P8, Fz, Cz, Pz, FC1, FC2, CP1, CP2, FC5, FC6, CP5, CP6, TP9 and TP10) using an MR-compatible electrode cap equipped with carbon wired $\mathrm{Ag} / \mathrm{AgCl}$ electrodes (Easycap, Herrsching-Breitbrunn, Germany). One additional electrode was placed under the right eye and vertical EOG was measured by calculating the bipolar derivation between Fp2 and this EOG channel, horizontal EOG was measured by calculating the bipolar derivation between F7 and F8. ECG was measured by two dedicated electrodes. One electrode was placed on the sternum and the other on the clavicle, near the shoulder. The ECG was calculated as the bipolar derivation between these two electrodes. A 250- $\mathrm{Hz}$ analog hardware filter was placed between the electrode cap and the EEG amplifier (Brainproducts, Munich, Germany). The EEG was recorded with a $10 \mathrm{~s}$ time constant and a $100 \mathrm{~Hz}$ low-pass filter and continuously sampled at $5 \mathrm{kHz}$. Impedances were kept under $5 \mathrm{k} \Omega$. All recordings were done with Brain Vision Recorder software (Brainproducts). EEG recordings outside the scanner were performed in the same way as inside the scanner, except that the sampling rate was $500 \mathrm{~Hz}$.

\section{Image acquisition}

MRI measurements were performed using a $1.5 \mathrm{~T}$ Sonata wholebody scanner (Siemens, Erlangen, Germany). Functional images were acquired using a gradient echo EPI sequence ( $T R=2.34 \mathrm{~s}$ including $50 \mathrm{~ms}$ dead time, TE $=30 \mathrm{~ms}, 33$ slices, $3 \mathrm{~mm}$ thickness with $0.5 \mathrm{~mm}$ gap, $\mathrm{FOV}=224 \mathrm{~mm}$, resulting in an isotropic voxel-size 3.5 by 3.5 by $3.5 \mathrm{~mm}$ ) A 3D MPRAGE pulse sequence was used for the anatomical scan.

\section{Analysis of EEG data}

The EEG data recorded inside the MR scanner were corrected for gradient and pulse artifacts along the lines described by Allen et al. $(1998,2000)$ using Vision Analyzer (Brainproducts). The major residual scan artifact is determined by how many slices are acquired in one second. Measuring 33 slices in 2.29 s resulted in a main residual artifact at $14.4 \mathrm{~Hz}$ and its higher harmonics, which spared the alpha $(8-12 \mathrm{~Hz})$ and theta $(4-7 \mathrm{~Hz})$ frequency bands that we were primarily interested in.

The data corrected for MR artifacts was subsequently low-pass filtered at $100 \mathrm{~Hz}$, downsampled to $500 \mathrm{~Hz}$ and re-referenced to a common average reference. The original reference electrode was recalculated as $\mathrm{FCZ}$, yielding a total of 30 EEG channels. In the subsequent step, residual eye blinks during the trial were corrected by applying the Gratton and Coles algorithm (Gratton et al., 1983). Subsequently, the trials were visually inspected for residual eye movements or other artifacts. Contaminated trials were subsequently excluded ( $9 \%$ of the trials on average).

Time-frequency analysis using a multitaper approach (Mitra and Pesaran, 1999) was carried out with the Fieldtrip toolbox for EEG/
MEG-analysis (FC Donders Centre for Cognitive Neuroimaging, Nijmegen, The Netherlands; see http://www.ru.nl/fcdonders/fieldtrip) running under Matlab (MathWorks, Natick, MA). The EEG power was analyzed from $1.25-20 \mathrm{~Hz}$ in $1.25 \mathrm{~Hz}$ steps for every $100 \mathrm{~ms}$. Multitapering was performed with a $800 \mathrm{~ms}$ time smoothing and $2.5 \mathrm{~Hz}$ frequency smoothing. Time-frequency analysis of higher frequency bands (up to $100 \mathrm{~Hz}$ ) indicated no observable WM modulations of power.

To reduce inter-subject variability and to normalize power changes across different frequency bands, power changes were calculated relative to a $0.5 \mathrm{~s}$ pre-trial baseline. The statistical significance of the WM load dependent power increases across the time frequency representations of these loads was evaluated by a cluster-based randomization procedure (Maris and Oostenveld, 2007). This test effectively controls the Type- 1 error rate in a situation involving multiple comparisons. This procedure allows for the use of user defined test statistics tailored to the effect of interest within the framework of a cluster-based randomization test. In our case we constructed a test statistic that reflected a parametric increase of power over WM loads.

First, for every electrode-time-frequency point the slope of the regression line fitted through the power values of the four loads was calculated. Subsequently, a one-tailed single sample t-test was performed on these slopes (giving uncorrected $p$-values). All data points that do not exceed a pre-set significance level (here 5\%) are zeroed. Clusters of adjacent non-zero data points in electrode-timefrequency space are computed, and for each cluster a cluster-level test statistic is calculated by taking the sum of all the individual $t$-statistics within that cluster. This statistic was entered in the cluster-based randomization procedure.

For comparison with the region of interest analysis of the fMRI data (see below) we also performed pair-wise comparisons for the average alpha and theta power during WM maintenance of the selected independent component (see below). For each subject we first averaged the relative power across all time steps in the maintenance interval for the frequency bin used for the construction of the EEGbased regressors. Subsequently we log transformed the EEG power data to make its distribution more Gaussian.

Since previous work showed the WM-related alpha power increase is mainly observed in the upper alpha band, we performed an analysis for the alpha sub-bands for both channel level data, as well as for the selected alpha independent component (see below). For channel level data we chose channel 02 , since the WM effect was maximal at this location. The alpha sub-bands were defined along the lines of Klimesch (1999). The individual alpha frequency (IAF) was determined by calculating the average power over all channels and over the $10 \mathrm{~s}$ spanning both the encoding and maintenance intervals. This $10 \mathrm{~s}$ window was subdivided in 5 segments of $2 \mathrm{~s}$, resulting in a frequency resolution of $0.5 \mathrm{~Hz}$. IAF was defined as the frequency with the largest power in the $8-12 \mathrm{~Hz}$ range. Subsequently the power was estimated for each load. For this, the middle $6 \mathrm{~s}$ of the retention interval were divided in 3 segments of $2 \mathrm{~s}$, discarding the first and last $0.5 \mathrm{~s}$. The alpha sub-bands were defined relative to IAF as follows: the lower- 1 alpha band ranged from IAF- $4 \mathrm{~Hz}$ to IAF-2 Hz; the lower-2 alpha band ranged from IAF $-2 \mathrm{~Hz}$ to IAF; the upper band ranged from $\mathrm{IAF}$ to $\mathrm{IAF}+2 \mathrm{~Hz}$. The power values for each frequency were normalized by dividing the power estimates for each load by the average power over the four loads. For the statistical analysis, a linear trend was fitted through the power values of the four loads for each subject separately. An increase in power with load should result in a significant positive slope, which was tested in a single sample onetailed $t$-test. The false discovery rate (Benjamini and Hochberg, 1995) was used as a correction for multiple comparisons (FDR $=0.05$ ).

Except for the correction for MR gradient and pulse artifacts, preprocessing and time-frequency analysis for the data outside the scanner was the same as for inside the scanner. 
Construction of EEG-based alpha and theta regressors

Fig. 2 presents a flow-chart of the preprocessing steps involved in the construction of the EEG regressor. To get denoised trial-by-trial estimates of the observed increase in alpha and theta power during the maintenance interval (see Results) we applied infomax independent component analysis (ICA) (Bell and Sejnowski, 1995) as implemented in EEGLAB version 5.03 (Delorme and Makeig, 2004). To obtain a component that modeled the WM alpha effect we lowpass filtered under $25 \mathrm{~Hz}$ before applying ICA. For three subjects a 6$17 \mathrm{~Hz}$ band-pass filter was applied because none of the independent components showed clear increased activity in the alpha band during the maintenance interval when the low-pass filter of was applied. Frontal theta activity is in general less pronounced than the alpha

Filtered data corrected for MR artifacts
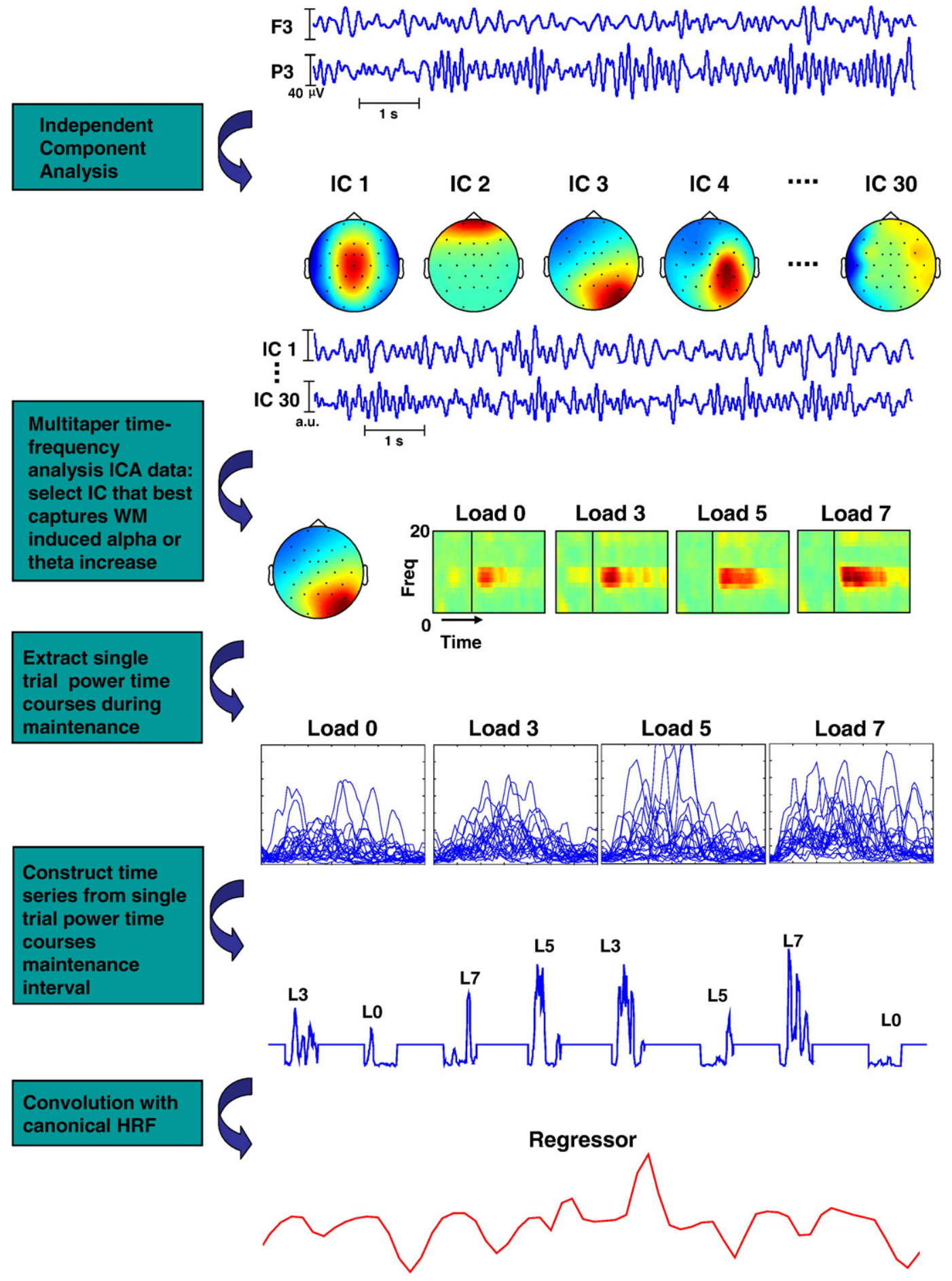

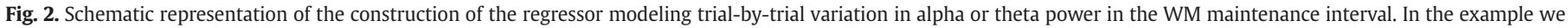

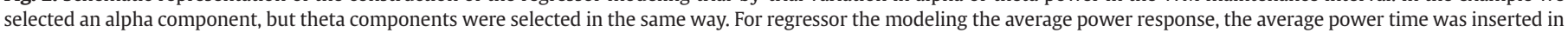
stead of the single trial power time courses. This procedure is described in more detail in the Methods section. 
activity, and therefore less likely to be separated from other sources and artifacts. For the construction of the theta regressor we therefore applied a 2-9 $\mathrm{Hz}$ band-pass filter prior to applying ICA to reduce the presence of other sources and artifacts in the analyzed data.

ICA was performed on the concatenated trials of all WM loads of all the three blocks. The trials started $2.5 \mathrm{~s}$ before onset of the encoding and ended $3 \mathrm{~s}$ after probe onset. A weight change of $10^{-7}$ was used as a stop criterion and the maximum number of iterations set at 512 .

The same multitaper technique with the same time and frequency smoothing as applied on the channel level EEG time series was also applied on the independent component-level time series. Subsequently the average time-frequency representation of power was calculated for each component for each WM load separately. For each subject one component for each frequency band was selected based on (i) the presence of an (parametric) average alpha or theta WM effect during the maintenance interval and (ii) a topography of the mixing weights that resembled the topography of the effects observed both inside and outside the scanner. In the situation where more than one component passed these criteria, we chose the component that explained the most variance in the channel level data (4 times for alpha, 2 times for theta). Significance of the alpha effect in the ICA data was assessed with the same cluster randomization technique as described above, except that only clustering in the time and frequency domains was used, since the selected component activity was a weighted mixture of all channels.

For the alpha effect, the peak frequency in the $7.5-13.75 \mathrm{~Hz}$ range of the selected IC was determined by calculating the average power over time and WM loads for each frequency bin during WM maintenance. The frequency with the highest average alpha power was selected to represent the trial-by-trial variation in alpha power, since the multitaper approach already integrated the power over a $5 \mathrm{~Hz}$ frequency range for each frequency step. The same procedure was followed for the theta effect in the $3.75-7.5 \mathrm{~Hz}$ range. For both frequency bands the individual average power time courses and the single trial power time courses during the maintenance interval of the selected component constitute the basis for two regressors.

To construct the first regressor, the power values across all trials and WM loads within one block of trials were first $z$-transformed. For the trials that were excluded at the artifact rejection stage, the average power response of that WM load was used. The $z$-transformed single trial power time series were then used to construct a time series that contained zeros outside the maintenance intervals. This time series was convolved with the canonical hemodynamic response function (HRF) from SPM5 (Wellcome Department of Imaging Neuroscience, London, UK; see http://www.fil.ion.ucl.ac.uk/spm) and resampled to obtain a single sample for every fMRI volume. This regressor was constructed for both the alpha and theta effect. Since these regressors reflect the variability of power across trials, they are termed TRL $\alpha$ and TRL $\theta$.

For the second regressor, the individual average power response across trials for each WM load was used instead of the single trial power time courses. Z-transformation, convolution and re-sampling was carried out in exactly the same way as for the first regressor. This regressor models the individual subjects' average alpha and theta response for each WM load and is therefore termed AVG $\alpha$ and AVG $\theta$ for alpha and theta respectively.

\section{FMRI model construction}

Preprocessing and analysis of the fMRI data was carried out in SPM5. The fMRI data was corrected for subject movement, corrected for differences in slice acquisition time, anatomically normalized to the canonical EPI template provided by SPM5 and smoothed with an isotropic Gaussian kernel of $10 \mathrm{~mm}$ full-width-at-half-maximum.

For the standard analysis a general linear model was constructed. Box-car functions convolved with the canonical HRF were used to model the encoding, retrieval and inter-trial intervals. Different func- tions were used for the different WM loads in the encoding and maintenance intervals. The retrieval interval was defined as the time between probe onset and the button press. The realignment parameters were added to control for movement related effects. Erroneous trials were modeled by separate regressors. The Load 3 versus Load 7 contrast was used to assess which regions were activated. An uncorrected voxel-level threshold of 0.001 was used with a cluster-level threshold of 0.05 corrected for multiple comparisons. This analysis was complemented with a ROI analysis over all the levels for the significant regions obtained in this contrast. Since the Load 3 versus Load 7 contrast already indicates a WM effect on BOLD, only repeated contrasts were computed (e.g. Load 0 vs. Load 3, Load 3 vs. Load 5 and Load 5 vs. Load 7) between the average regression coefficients (so called beta weights) related to the regressors of the four WM loads.

In the first model that included EEG regressors (Model I) the boxcar regressors modeling the maintenance interval were replaced by either TRL $\alpha$ or TRL $\theta$. All the other stages of the trial were still modeled with HRF convolved box-car functions. The realignment parameters were again added to control for movement related effects. Second level single sample $t$-tests were used to test for regions showing a significant relation to TRL $\alpha$ and TRL $\theta$. Significant clusters were identified as those clusters passing an uncorrected voxel-level threshold of 0.001 , and a corrected cluster-level threshold of 0.05 (Gaussian random field correction).

The explained variance in the TRL $\alpha / \theta$ regressor Model I is a sum of variances that could also be related to (1) box-car regressors modeling the maintenance interval, (2) the average individual power time course for each WM load and (3) the trial-by-trial variation in power. A Gram-Schmidt orthogonalization procedure was followed (for similar use see Eichele et al., 2005) to obtain uncorrelated regressors that model these three sources of variance. The regressors included in the design matrix of Model II consists of (1) HRF convolved box-car functions for all the different stages of the trial, (2) the AVG $\alpha / \theta$ regressors orthogonalized to these box-car regressors and (3) TRL $\alpha / \theta$ regressors orthogonalized to both box-car regressors and AVG $\alpha / \theta$ regressors. The realignment parameters were also included. The clusters where the BOLD signal was found to be related to single trial alpha and theta estimates in Model I were used as the templates for regions of interest (ROI) analyses on which the analyses in Model II were based. All effects were tested on group level and based on the beta weights associated with the regressors introduced here.

If a region was functionally related to the observed WM-induced theta or alpha increases, it should in theory show an effect when analyzed with all three orthogonalized sets of regressors. That is, the regions should show a parametric BOLD response (as analyzed by the box-car regressors) that is in line with the parametric increase in alpha or theta power. If we assume that the average deviation from the box-car response was reflected in the BOLD signal and there was variation in the strength of the response over trials that was also related to the BOLD signal, these regions should show a significant relation to the orthogonalized AVG and TRL regressors respectively. The parametric WM effects for the ROIs were tested for a linear trend over loads, followed up with repeated contrasts between the levels to indicate where and how the pattern deviated from a linear increase with load. The significance of the relation between the ROIs and the orthogonalized $\mathrm{AVG} \alpha / \theta$ and orthogonalized TRL $\alpha / \theta$ was then assessed by single sample two sided $t$-tests. If the BOLD response in a region is related to WM-induced power increases, the sign of this relation should be consistent for the regressors modeling the three sources of variance. This means that a positive parametric BOLD effect observed in a region should go together with a positive relation between BOLD and the orthogonalized AVG and TRL regressor.

In practice it was found that the orthogonalized AVG regressor can lack sensitivity and statistical power. Since the WM-related alpha effect decreased during the maintenance interval, the AVG $\alpha$ also 
A

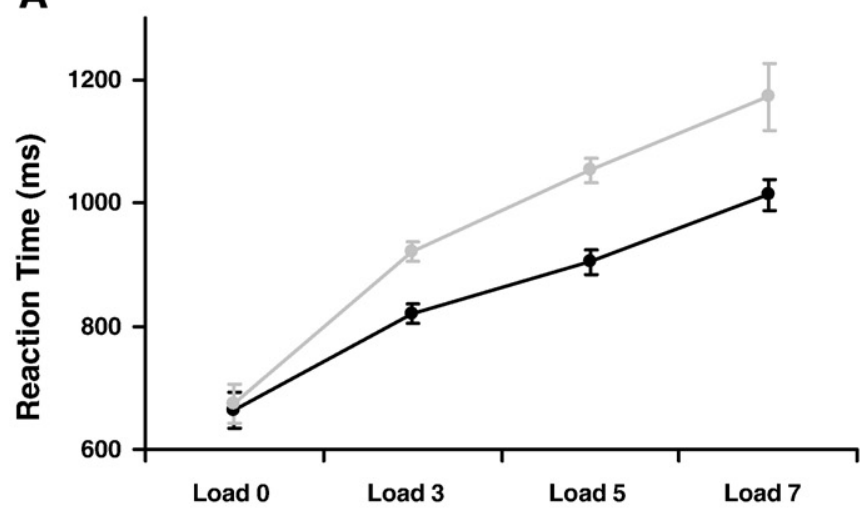

B

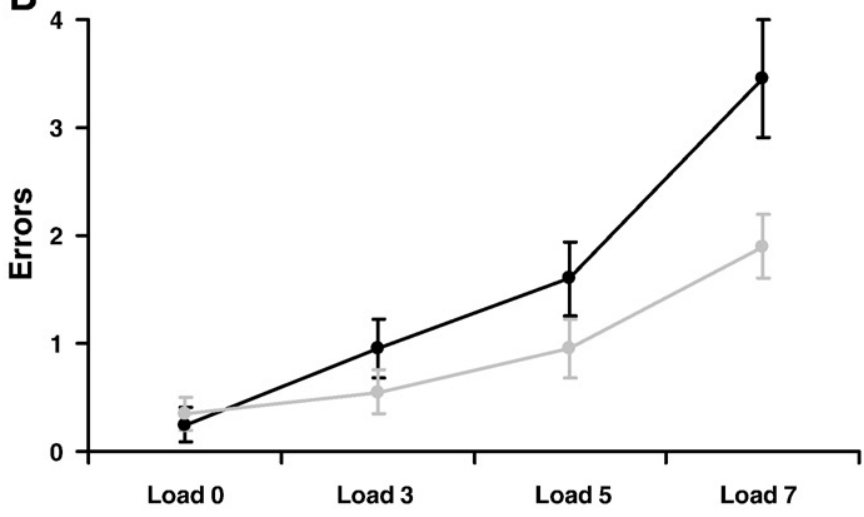

WM Load

Match/Index finger response required

Mismatch/Middle finger response required

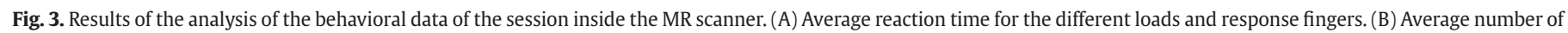
errors for the different loads and response fingers. Error bars indicate the standard error.

Outside MR: Channel Data

A

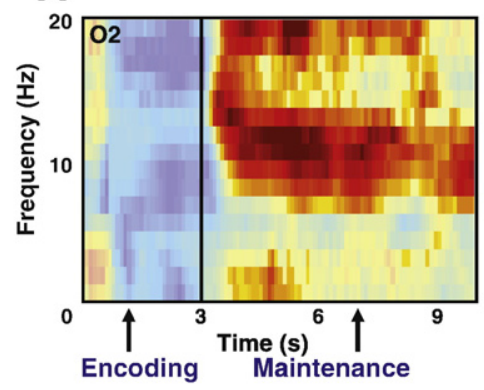

D
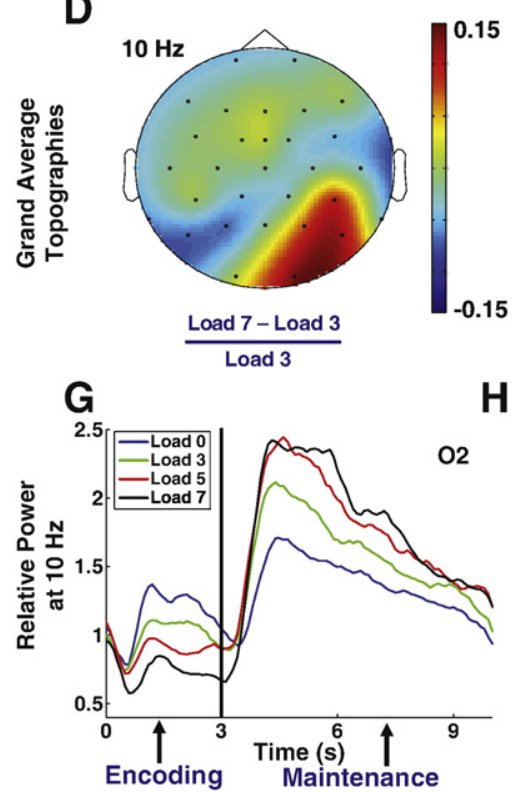

Inside MR: Channel Data

B

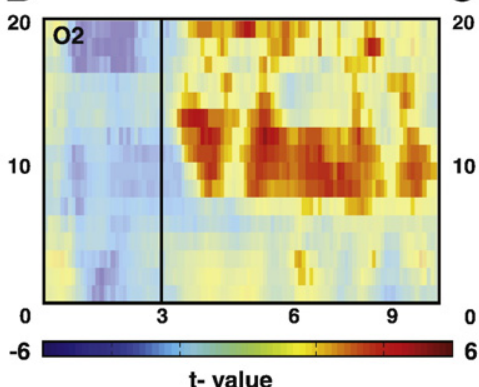

$E$

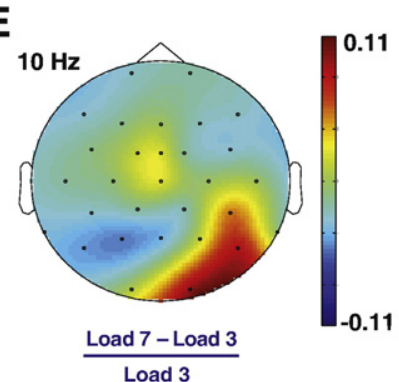

H

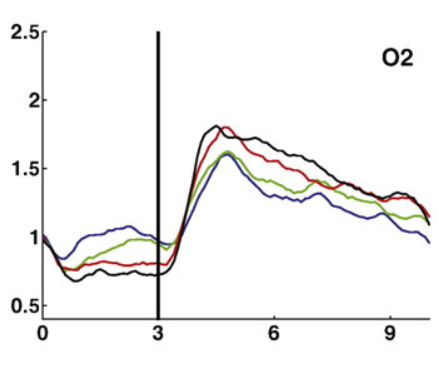

Inside MR: Selected IC

C

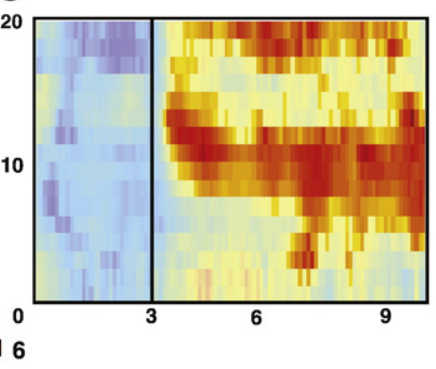

$F$

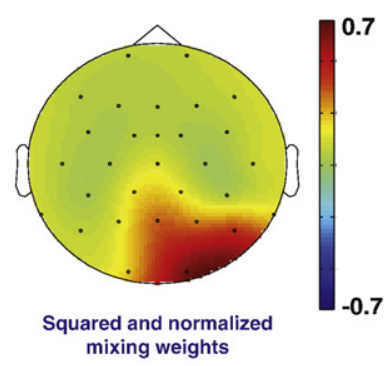

I

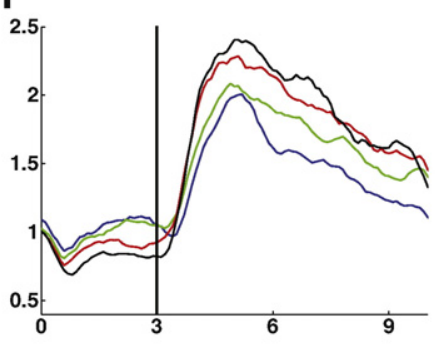

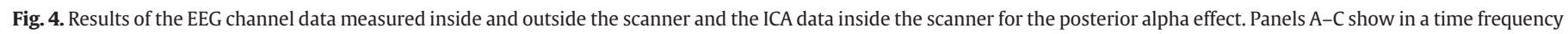

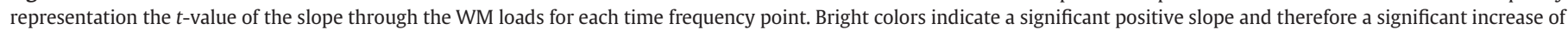

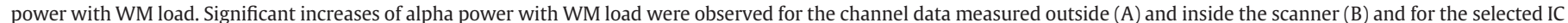

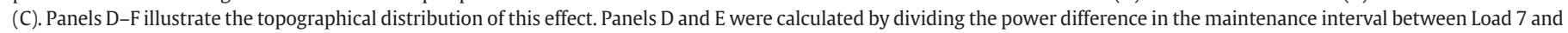

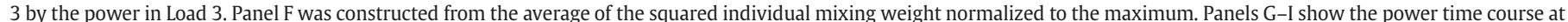

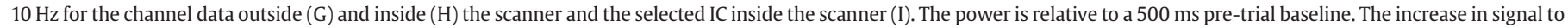

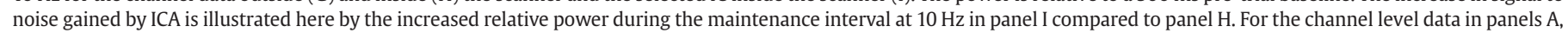
B. $\mathrm{G}$ and $\mathrm{H}$ channel $\mathrm{O} 2$ is shown. 
mostly models decreases, while the average theta response after convolution with the HRF does not deviate much from the HRF convolved box-cars. We therefore consider regions to be functionally related to the WM load-related alpha and theta power increases if they show a significant parametric BOLD modulation with WM load, as well as a significant relation with the orthogonalized TRL $\alpha / \theta$ regressors.

\section{Results}

Behavioral data

Analysis of variance of the reaction time data reveal significant main effects for the factors Load $(F(3,57)=48.14, p<0.001)$ and Response Finger (Match and Mismatch) $(F(1,19)=18.40, p<0.001)$. A significant Load by Response Finger $(F(3,57)=4.26, p<0.05)$ interaction effect is observed.

The main effect of Load is related to an increase of reaction time with WM load. (linear contrast: $(F(1,19)=59.97, p<0.001)$ The main effect of Response Finger is related to a faster response to match/index finger responses than to mismatch/middle finger responses. The Load by Response Finger interaction effect indicates that the difference is only present in the Load 3, Load 5 and Load 7 conditions. Simple main effects also indicate this (see Fig. 3A). The difference is not significant for Load $0(t(19)=0.78$, not significant $)$ but is significant for Load $3(t$ $(19)=4.58, p<0.001)$, Load $5(t(19)=4.71, p<0.001)$ and Load $7(t(19)=$ $2.511, p<0.05)$. These results are in line with previous behavioral results on Sternberg WM tasks.

Fig. 3B shows that the number of errors significantly increased with WM load $(F(3,57)=17.36, p<0.001)$, and significantly more errors were made in the match than mismatch condition $(F(1,19)=6.04$, $p<0.05)$. A significant linear trend is observed for the increase in the number of errors with load $(F(1.19)=31.68, p<0.001)$. Fig. 3B suggests the difference in the number of errors between match and mismatch trials might have increased with load. However, the Load by Response interaction effect shows this effect is only marginally significant $(F$ $(3,57)=2.93, p=0.07)$. Simple main effects however reveal significantly more errors for Match trials in load $7(t(19)=2.35, p<0.01)$, but not in the other load conditions (Load 0: $t(19)=-0.57$, not significant; Load 3: $t(19)=1.57$, not significant; Load 5: $t(19)=1.39$, not significant).

\section{EEG data}

Fig. 4 shows a parametric increase of alpha power with WM load during the maintenance interval on right posterior electrodes for the data outside the scanner, for the data recorded inside the scanner and for the selected independent components from the data inside the

\section{Channel level: 02}

\section{A}

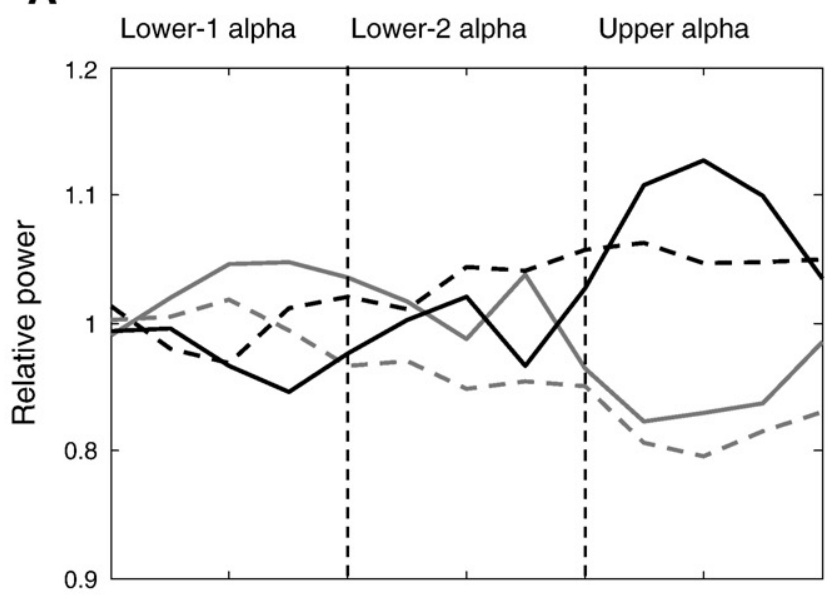

$$
\text { C }
$$

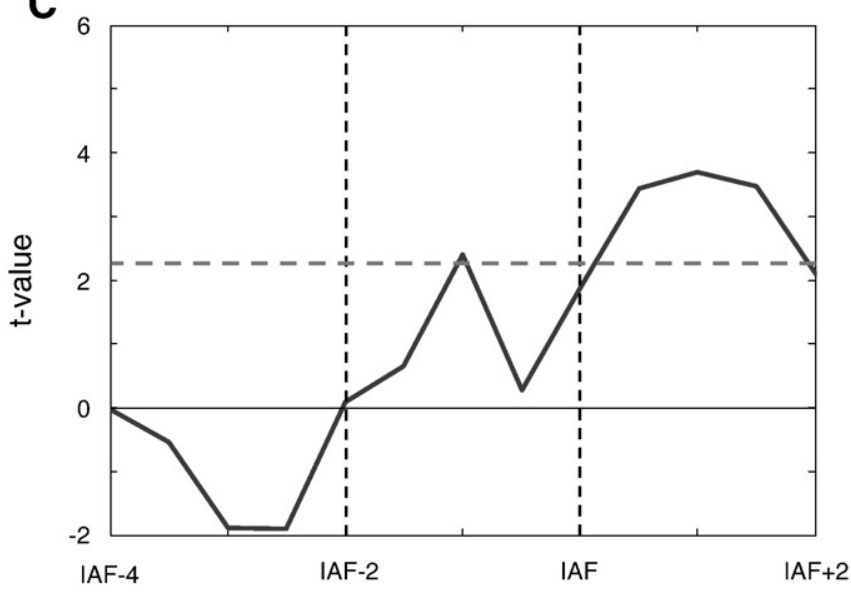

\section{ICA: Selected alpha component}

B

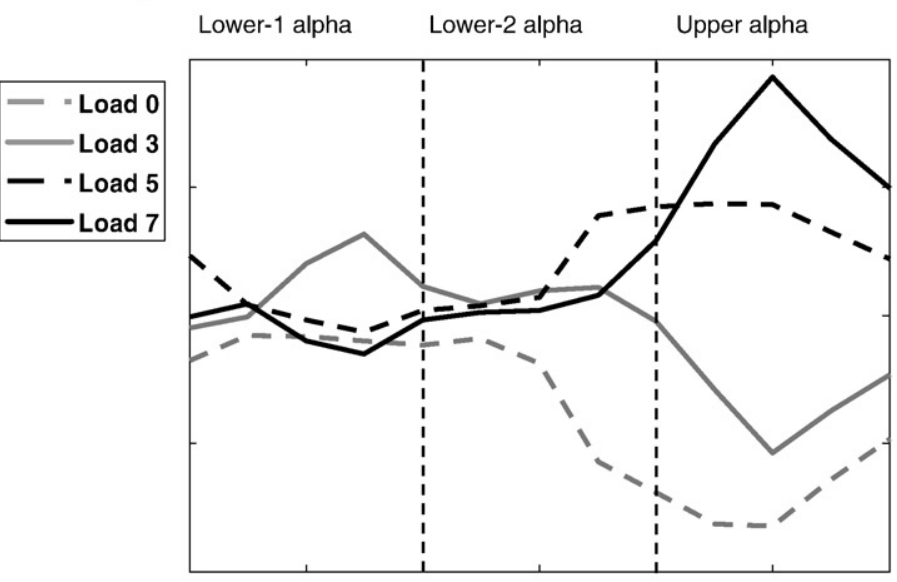

D

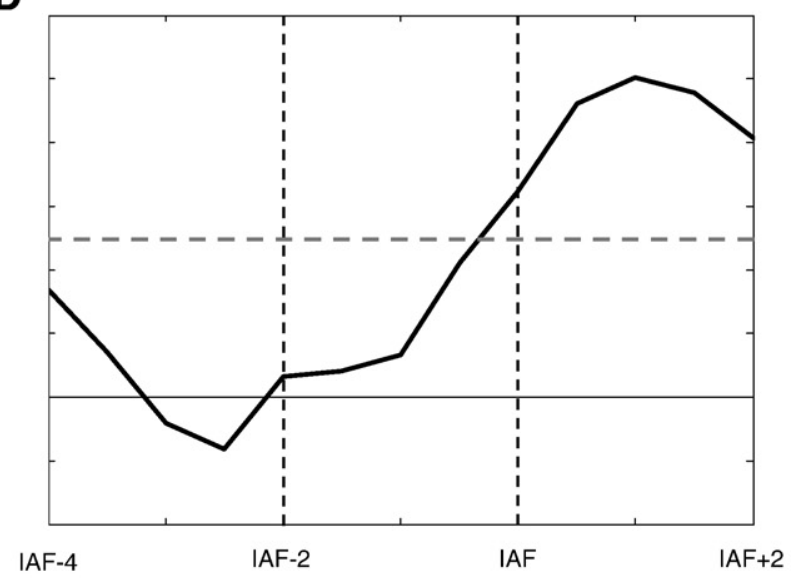

Relative alpha frequency $(\mathrm{Hz})$

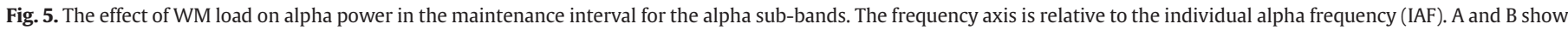

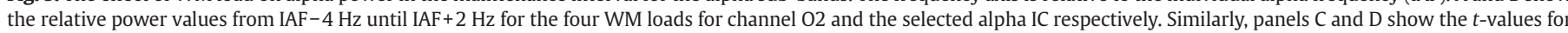

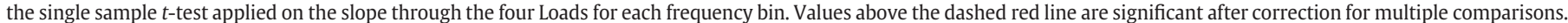



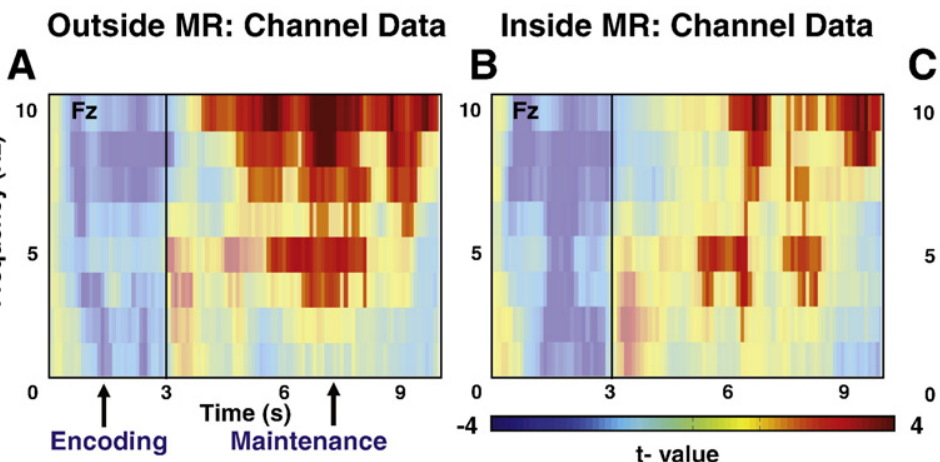

\section{Inside MR: Selected IC}

C

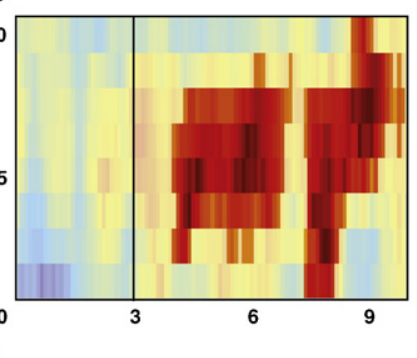

\section{D}
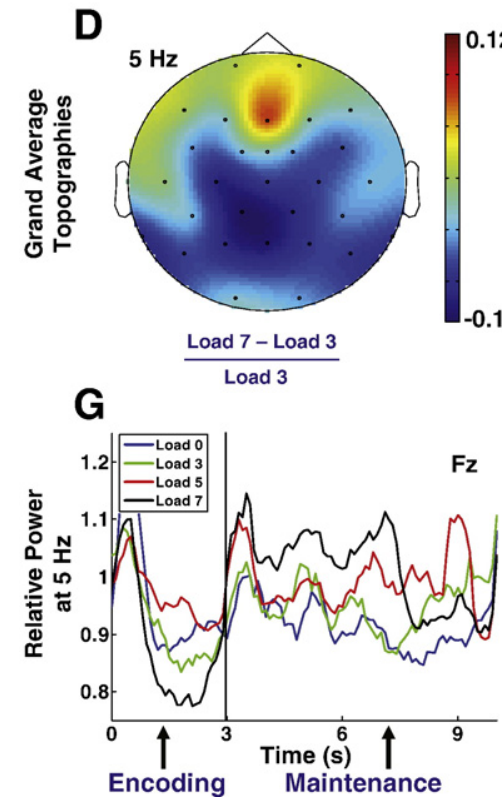

E

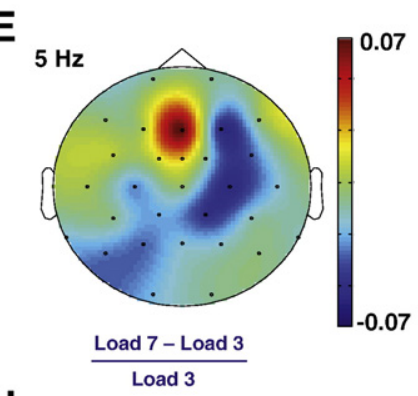

H

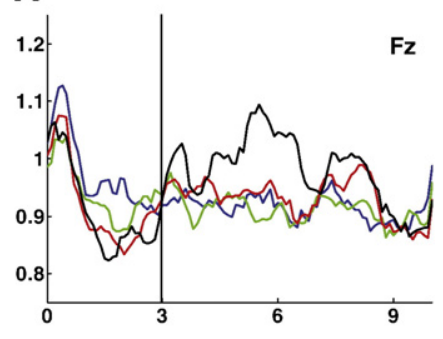

$\mathbf{F}$

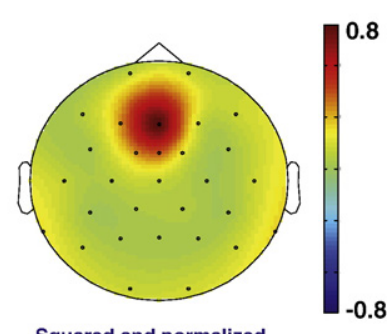

Squared and normalized mixing weights

|

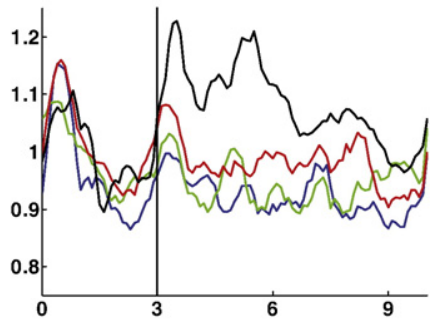

Fig. 6. Similar as Fig. 4, but then for the frontal theta effect. Here ICA not only increased the relative power, but also the extent of the significant WM effect in panel C.

scanner. The results displayed in first two columns of Fig. 4 indicate that inside and outside the MR scanner qualitatively the same parametric alpha increase was measured. Figs. $4 \mathrm{H}$ and I illustrate a clear increase in the relative power during WM maintenance after ICA. This indicates that ICA separates the right posterior alpha effect from other components that contributed to this frequency band, and as a consequence increases the signal to noise ratio. Pair-wise comparisons for the average alpha power during WM maintenance reveal significant differences between Load 3 and Load 0 and between Load 5 and Load 3, but not between Load 7 and Load 5 (see Fig. 9I). The analysis for the different alpha sub-bands reveals, that both for channel level and the IC data, the increase of alpha power with load can mainly be attributed to the effect in the upper alpha band (see Fig. 5). The average individual alpha frequency is observed at $9.5 \mathrm{~Hz}(\mathrm{SD}=0.8 \mathrm{~Hz})$.
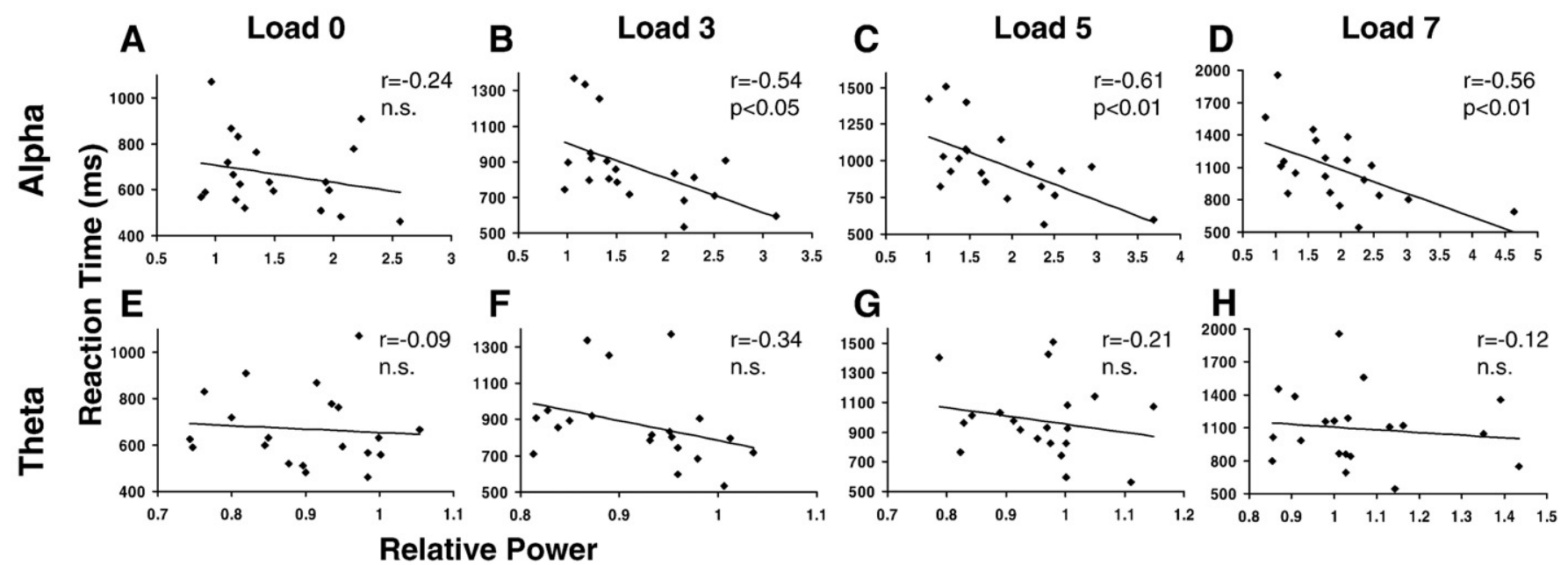

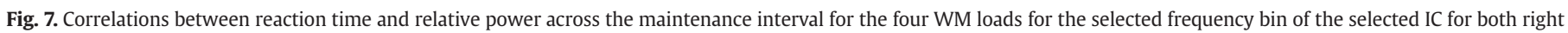
posterior alpha (A-D) and frontal theta (E-H). 
Table 1

Regions showing a significant WM modulation in the Load 7 versus Load 3 contrast

\begin{tabular}{|c|c|c|c|c|c|c|}
\hline \multirow[t]{2}{*}{ Brodman area } & \multirow[t]{2}{*}{ Anatomical structure } & \multirow[t]{2}{*}{ Size (voxels) } & \multicolumn{3}{|c|}{ MNI coordinates } & \multirow[t]{2}{*}{$t$-value } \\
\hline & & & $\bar{x}$ & $y$ & $z$ & \\
\hline \multicolumn{7}{|l|}{ Positive effects } \\
\hline $44 / 45 / 9 / 6$ & Broca's region/left inferior frontal gyrus/Insula & 3012 & -48 & 18 & 28 & 6.71 \\
\hline $8 / 6$ & Left superior medial frontal gyrus/pre-SMA & 932 & -2 & 20 & 50 & 5.78 \\
\hline \multirow[t]{2}{*}{$9 / 46$} & Right dorsolateral prefrontal cortex & 507 & 46 & 42 & 26 & 5.53 \\
\hline & Right insula & 250 & 34 & 24 & -4 & 4.73 \\
\hline 7 & Left superior parietal cortex & 244 & -48 & -42 & 54 & 4.37 \\
\hline \multicolumn{7}{|l|}{ Negative effects } \\
\hline $23 / 31 / 17 / 18 / 19 / 37 / 38 / 39$ & Various regions in parietal and occipital cortices & 15,841 & 8 & -48 & 30 & 7.02 \\
\hline $9 / 10 / 32 / 12$ & Medial frontal cortex & 3029 & 0 & 62 & -4 & 5.61 \\
\hline 21 & Left middle temporal gyrus & 397 & -60 & 2 & -24 & 4.05 \\
\hline
\end{tabular}

Fig. 6 shows the results for the frontal theta effect in a similar way as Fig. 4 does for right posterior alpha. Also the frontal theta effect can be observed outside as well as inside the scanner. The topographies clearly show the mid-frontal distribution associated with frontal theta (see Makeig et al., 2002; Onton et al., 2005). Applying ICA again increases the signal to noise ratio as expressed in a relative power increase. In this case ICA also leads to a better contrast between the four loads (see Fig. 6I). Pairwise comparisons for the average theta power during WM maintenance reveal significant differences between Load 5 and Load 3 and between Load 7 and Load 5, but not between Load 3 and Load 0 (see Fig. 10I).

For the EEG data recorded inside the scanner correlations were computed between reaction time and the average relative alpha and theta power during the maintenance interval for the selected independent component (see Fig. 7). This was done for each WM load separately. Significant (two sided) negative correlations between reaction time and alpha power in the maintenance interval are observed for Load 3, 5 and 7. For theta power no significant correlations with reaction time were observed.

\section{FMRI data}

\section{Conventional fMRI analysis}

The Load 7 versus Load 3 contrast shows significant clusters of activity in the right dorsolateral prefrontal cortex (DLPFC), medial

\section{A}

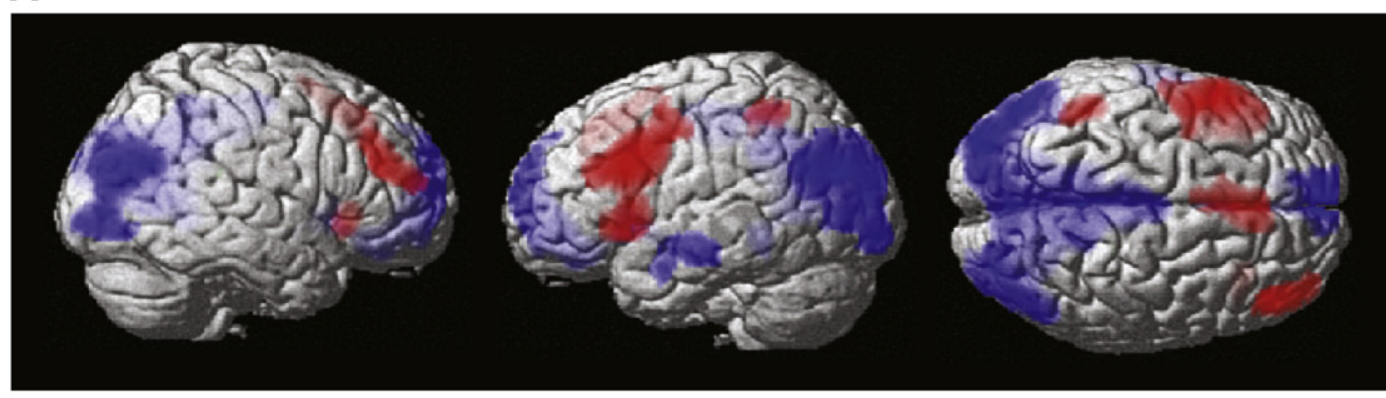

B

Activations Deactivations
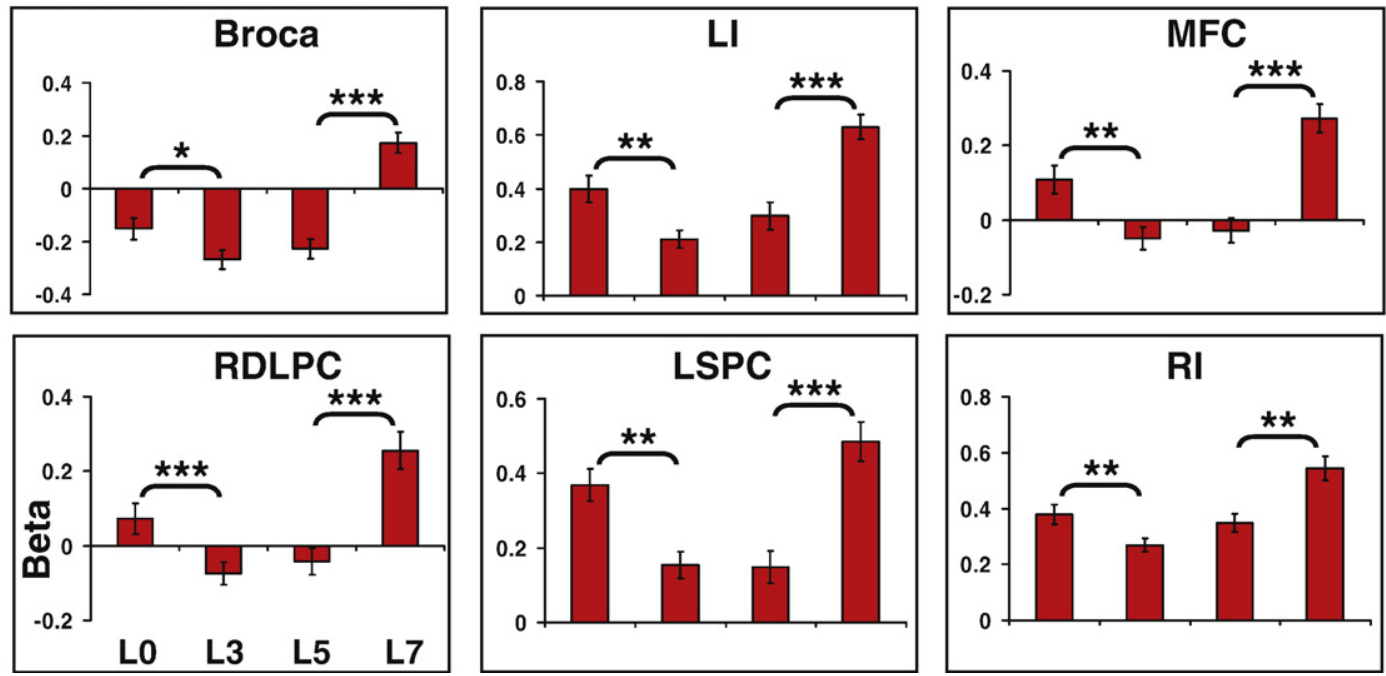

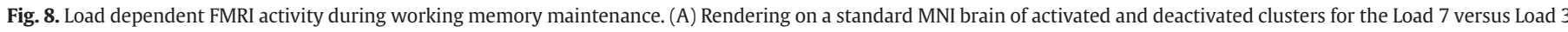

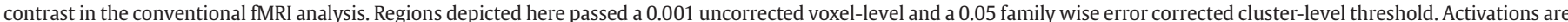

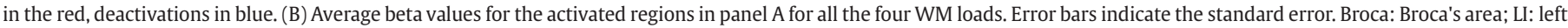

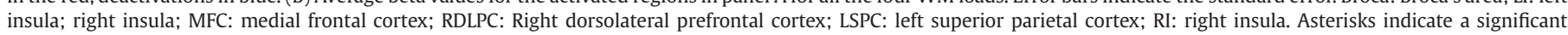
difference as tested with the repeated contrasts in the ROI analysis. ${ }^{*}|t(19)|>2.093, p<0.05 ; * *|t(19)|>2.861, p<0.01 ; * * *|t(19)|>3.883, p<0.001$. 
frontal cortex, left inferior parietal lobule and a left lateralized cluster spanning the left ventrolateral frontal cortex, a part of Broca's region and the insula (see Table 1 and Fig. 8). At a higher voxel-level threshold ( $p=0.0001$, uncorrected for multiple comparisons) this cluster splits in two clusters (results not shown) that are significant at cluster level (corrected for multiple comparisons). The first cluster is mainly located in Broca's region (BA 44 and BA 45), but also stretched into to more dorsolateral areas (BA9 and BA 6). The second cluster is inferior to the first, mainly in the left insula. Negative effects are present in various regions in parietal, occipital cortices, left temporal and medial frontal regions. ROI analysis (see Fig. 8B) reveal that for all these regions activity is higher in the Load 0 than in the Load 3 condition. From Load 3 to Load 7 a monotonic increase in activity with WM load is observed in all regions except the superior parietal cortex, although only the differences between Load 5 and 7 are significant.

\section{Integrated EEG/fMRI analysis}

\section{Alpha}

Table 2 lists the clusters where the BOLD signal show a significant negative relation with TRL $\alpha$ in model I, where the single trial alpha power time courses model the maintenance interval (Fig. 9A). No positive clusters are observed. The linear trend analysis for the ROIs in Model II (Figs. 9C-E) reveal significant decreases in BOLD signal with WM load for the right middle temporal gyrus $(F(1,19)=43.59$, $p<0.001)$, primary visual cortex: $(F(1,19)=22.03, p<0.001)$ left cerebellum $(F(1,19)=33.79, p<0.001)$ left supramarginal gyrus $(F(1,19)=$ $20.11, p<0.001)$ right supramarginal gyrus $(F(1,19)=34.92, p<0.001)$. No significant linear trend is observed for the right middle frontal gyrus: $(F(1,19)=0.01$, not significant). Pair-wise comparisons however reveal that only the primary visual cortex and the posterior part of the right middle temporal gyrus show a pattern of decreased activity that is in line with the pattern of increase in alpha power (Figs. 9C and 9I). The cerebellum shows the same pattern for the lowest three loads, but shows a non significant increase in Load 7 compared to Load 5. Other regions show no significant load dependent decreases in activity beyond Load 3. ROI analysis for orthogonalized AVG $\alpha$ regressor reveal significant negative correlations with the right middle temporal gyrus, primary visual cortex, and the left cerebellum (see Fig. 9D) For TRL $\alpha$ orth the ROI analysis reveals that all regions show a significant negative correlation with this regressor (see Fig. 9F). In summary, the right middle temporal cortex and the primary visual cortex show both a parametric decrease with WM load that is in line with the frontal theta increase and a negative correlation with TRL $\alpha$-orth. We therefore conclude that load dependent BOLD decreases in these regions are related to the load dependent increases in alpha power (see Fig. 9B).

\section{Theta}

In contrast to the correlation to alpha power, negative as well as positive correlations with TRL $\theta$ are observed in model I (see Table 3 and Fig. 10A). For the regions where BOLD correlates positively with TRL $\theta$ in model I, the ROI analyses in Model II reveal that there are no significant linear treds observed for the left $(F(1,19)=0.12$, not significant.) and right $(F(1,19)=2.61$, not significant $)$ inferior frontal gyrus. Both these regions do not show a significant relation (see Fig. 10D) with orthogonalized AVG $\theta$ but do show a significant positive relation with orthogonalized TRL $\theta$ (see Fig. 10E).

The regions where BOLD correlates negatively with TRL $\theta$ in Model I do show an significant linear decrease with WM load (medial prefrontal cortex: $F(1,19)=15.08, p<0.01$; posterior cingulate cortex $F$ $(1,19)=35.59, p<0.001$; left angular gyrus: $F(1,19)=34.27, p<0.001$; right angular gyrus: $F(1,19)=21.48, p<0.001)$. Except for the right angular gyrus, these regions show a monotonic decrease with WM load that is strongest for the Load 7 condition (Fig. 10C). This strong decrease in activity in Load 7 is in line with the strong increase observed in frontal theta power (Fig. 10I). ROI analyses for orthogonalized AVG0 reveal only a significant negative correlation with the medial prefrontal cortex (see Fig. 10D). All selected regions show a significant negative relation with orthogonalized TRL $\theta$ (see Fig. 10E). In summary, the medial prefrontal cortex, posterior cingulate/precuneus and the left angular gyrus show both a parametric decrease with WM load that is in line with the increase in frontal theta power and a negative correlation with TRL $\theta$-orth. We therefore conclude that load dependent BOLD decreases in these regions are related to the load dependent increases in theta power (see Fig. 10B).

\section{Discussion}

In the present study, we used simultaneously recorded EEG and fMRI to investigate if the brain regions related to WM maintenance by previous fMRI studies are also functionally related to WMinduced posterior alpha and frontal theta increases. Analysis of the EEG data inside and outside the MR scanner revealed parametric increases with WM load in right posterior alpha and frontal theta power. Conventional analysis of the fMRI data yielded an increase in activity from Load 3 to Load 7 in a set of regions that have been related to verbal WM tasks (Cabeza and Nyberg, 2000). In the Load 0 condition these regions also showed increased activity compared to Load 3, indicating this condition did not function well as a high level baseline. The integrated single trial EEG/fMRI analysis revealed a functional relation between WM-induced right posterior alpha increases and BOLD decreases in right middle temporal gyrus and primary visual cortex. The WM-induced frontal theta power was found to be related to decreased activity in a set of regions that together form the default mode network (DMN; Gusnard and Raichle, 2001; Raichle et al., 2001; Shulman et al., 1997). The results of this integrated analysis are discussed in more detail below.

\section{Right posterior alpha-BOLD correlations}

The right posterior alpha increase with WM load is in line with earlier results obtained by Jensen et al. (2002) and Tuladhar et al. (2007). Interestingly, Jensen et al. (2002), who also used a verbal WM task, also reported a predominantly right lateralized effect, although

Table 2

Relation between the BOLD signal and right posterior alpha activity

\begin{tabular}{|c|c|c|c|c|c|c|c|c|c|}
\hline \multicolumn{7}{|l|}{ Model I } & \multicolumn{3}{|l|}{ Model II } \\
\hline \multirow{2}{*}{$\begin{array}{l}\text { Brodman } \\
\text { area }\end{array}$} & \multirow[t]{2}{*}{ Anatomical structure } & \multirow[t]{2}{*}{ Size (voxels) } & \multicolumn{3}{|c|}{ MNI coordinates } & \multirow[t]{2}{*}{$t$-value } & \multirow[t]{2}{*}{ Param. WM } & \multirow[t]{2}{*}{ Orth. AVG $\alpha$} & \multirow[t]{2}{*}{ Orth. TRL $\alpha$} \\
\hline & & & $\bar{x}$ & $y$ & $z$ & & & & \\
\hline \multicolumn{10}{|c|}{ Negative effects } \\
\hline $37 / 21 / 19$ & Right middle temporal gyrus & 2002 & 62 & -48 & 10 & 8.28 & + & + & + \\
\hline $9 / 46 / 6$ & Right middle frontal gyrus & 1621 & 38 & 38 & 20 & 6.91 & & & + \\
\hline \multirow[t]{2}{*}{17} & Left and right primary visual cortex & 1100 & 14 & -90 & 10 & 5.72 & + & + & + \\
\hline & Left cerebellum/left middle occipital gyrus & 654 & -30 & -70 & 8 & 5.14 & & + & + \\
\hline 40 & Left supra marginal gyrus & 228 & -52 & -46 & 38 & 5.52 & & & + \\
\hline 40 & Right supra marginal gyrus & 210 & 58 & -38 & 38 & 5.20 & & & + \\
\hline
\end{tabular}

+Regions that show a parametric modulation with WM load, a significant correlation to the orthogonalized AVG $\alpha$ regressor or orthogonalized TRL $\alpha$ in Model II. 


\section{A Correlations with alpha in Model I}

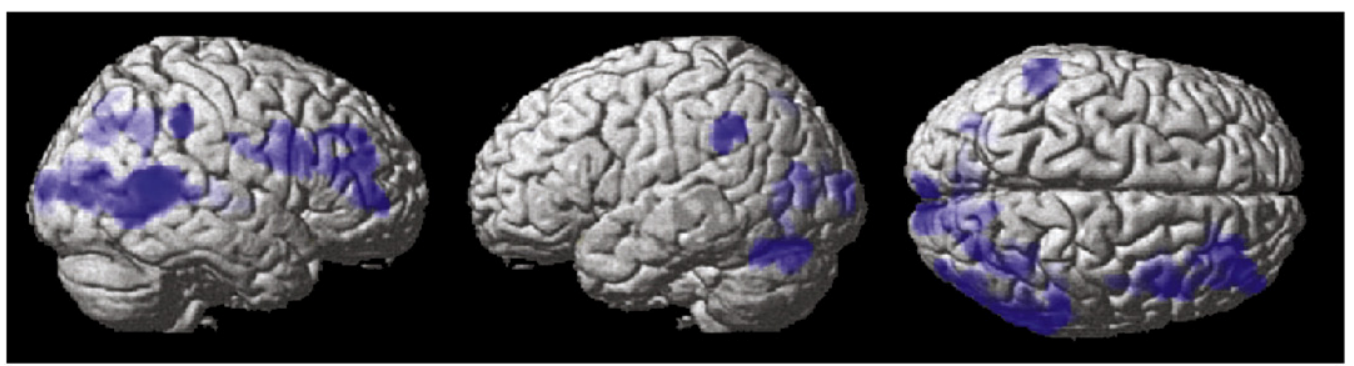

B

Regions related to WM alpha increase

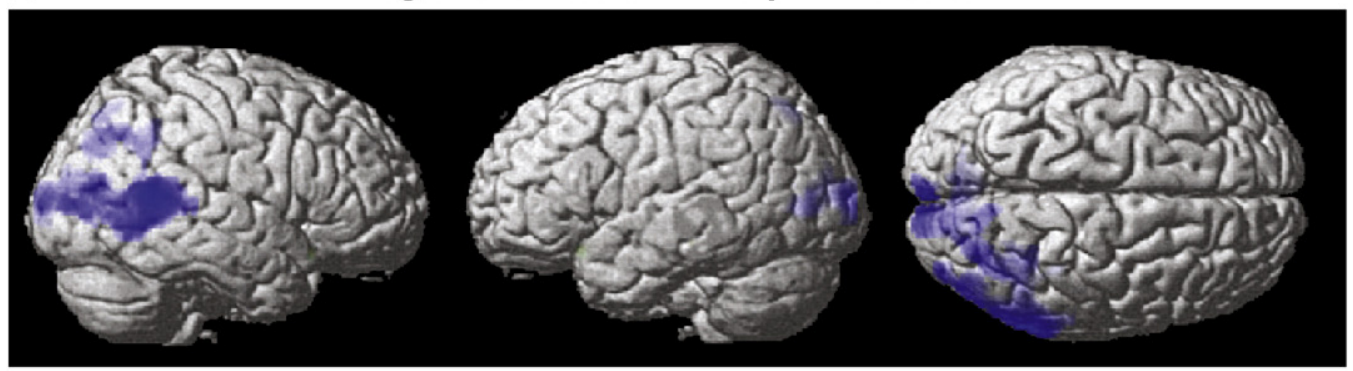

Negative correlations/deactivations

C
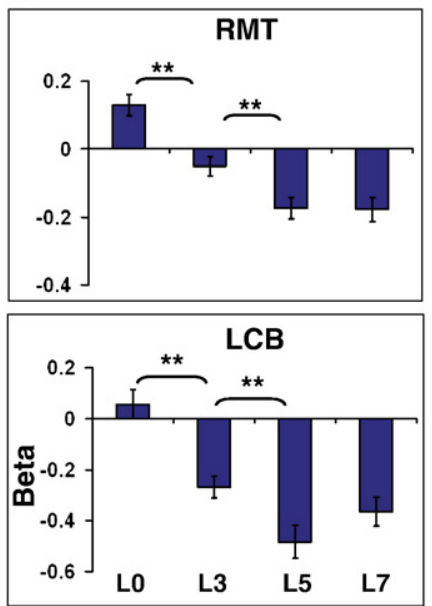

D
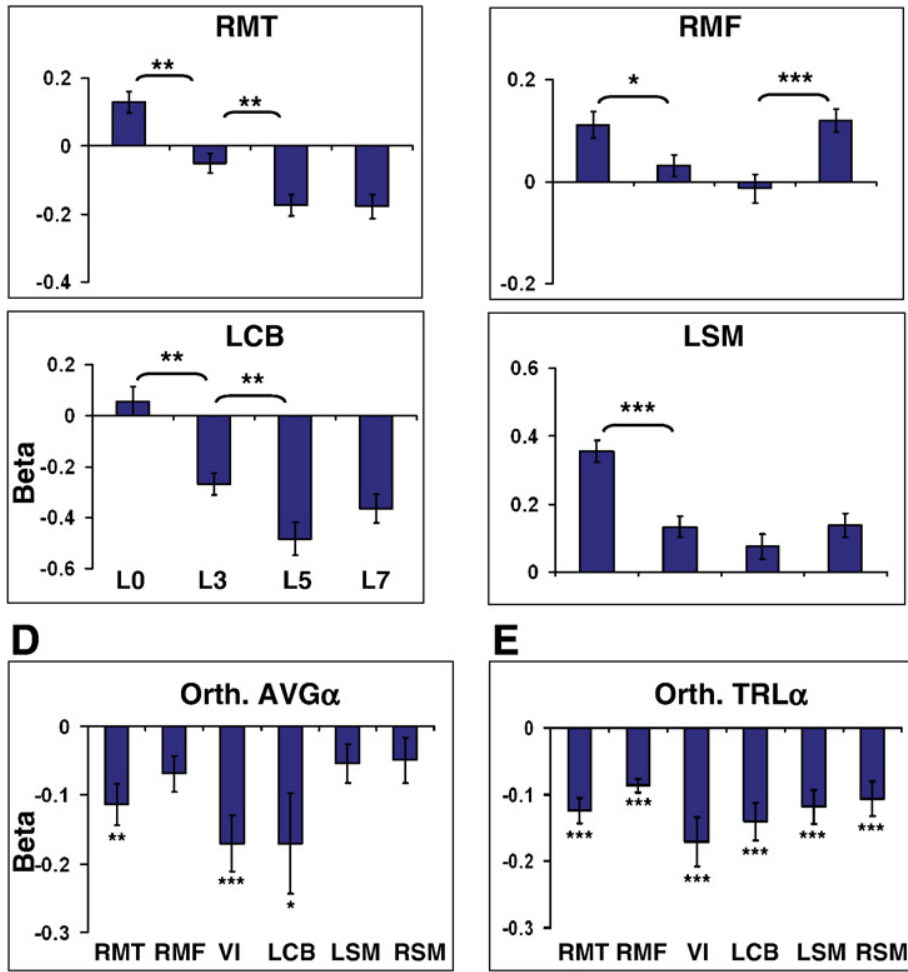

E

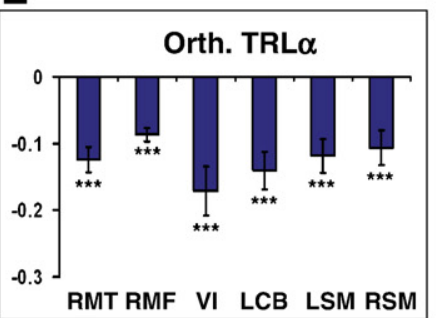

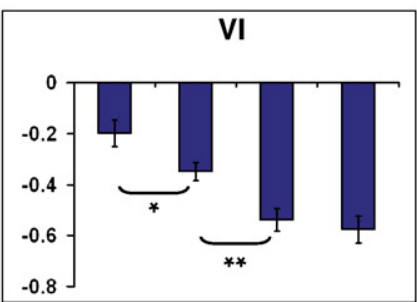

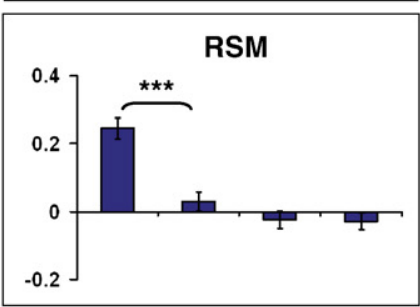

$\mathbf{F}$

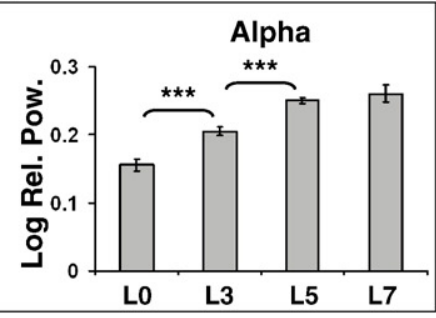

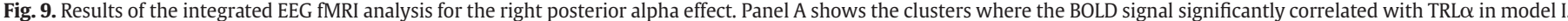

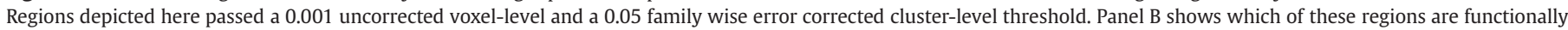

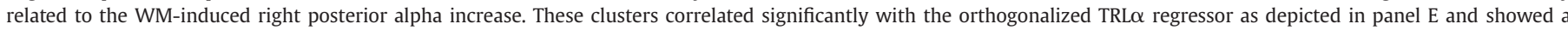

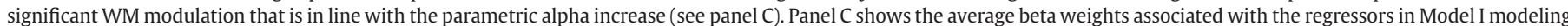

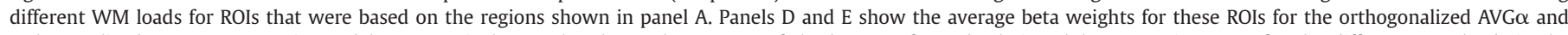

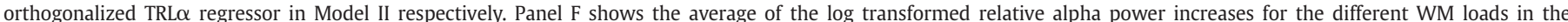

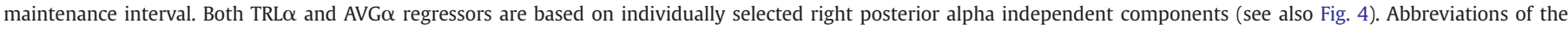

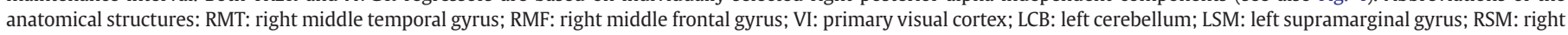
supramarginal gyrus. ${ }^{*}|t(19)|>2.093, p<0.05 ; * *|t(19)|>2.861, p<0.01 ; * * *|t(19)|>3.883, p<0.001$. Errors bars indicate the standard error. 
Table 3

Relation between the BOLD signal and frontal theta activity

\begin{tabular}{|c|c|c|c|c|c|c|c|c|c|}
\hline \multicolumn{7}{|l|}{ Model I } & \multicolumn{3}{|l|}{ Model II } \\
\hline \multirow{2}{*}{$\begin{array}{l}\text { Brodman } \\
\text { area }\end{array}$} & \multirow[t]{2}{*}{ Anatomical structure } & \multirow{2}{*}{$\begin{array}{l}\text { Size } \\
\text { (voxels) }\end{array}$} & \multicolumn{3}{|c|}{ MNI coordinates } & \multirow[t]{2}{*}{$t$-value } & \multirow[t]{2}{*}{ Param. WM } & \multirow[t]{2}{*}{ Orth. AVG $\theta$} & \multirow[t]{2}{*}{ Orth. TRL $\theta$} \\
\hline & & & $\bar{x}$ & $y$ & $z$ & & & & \\
\hline \multicolumn{10}{|c|}{ Positive effects } \\
\hline 43 & Left inferior frontal gyrus & 272 & -64 & -4 & 14 & 6.23 & & & + \\
\hline 43 & Right inferior frontal gyrus & 250 & 58 & 0 & 8 & 5.34 & & & + \\
\hline \multicolumn{10}{|c|}{ Negative effects } \\
\hline $9 / 10 / 32$ & Medial prefrontal cortex & 3772 & 6 & 60 & 32 & 6.82 & + & + & + \\
\hline $23 / 31$ & Posterior cingulate cortex/precuneus & 970 & -2 & -52 & 28 & 6.60 & + & & + \\
\hline 39 & Left angular gyrus & 420 & -52 & -68 & 38 & 5.75 & + & & + \\
\hline 39 & Right angular gyrus & 157 & 54 & -60 & 42 & 5.51 & & & + \\
\hline
\end{tabular}

+Regions that show a significant relation to the three sources of variance in Model II that is in line with the sign of the correlation with TRL $\theta$.

their effect seems to have a more anterior scalp distribution than the effect we observed.

We found a variety of regions, predominantly located in the right hemisphere, where the BOLD signal correlated negatively with the right posterior alpha power increase (TRL $\alpha$ ). Of those regions, only the right primary visual cortex (BA17) and the right middle temporal gyrus (BA 37) showed a significant relation with the three sources of variance that is in line with this monotonic increase in alpha power. For these two regions, a monotonic decrease in the BOLD signal with WM load as well as negative correlations with orthogonalized AVG $\alpha$ and orthogonalized TRL $\alpha$ regressors were observed. We therefore conclude these regions and possibly also the left cerebellum, where only the Load 7 condition showed a deviation from the expected pattern, were functionally related to the observed WM-induced alpha power increase.

For the other regions that correlated with alpha power fluctuations there is less evidence that they were functionally related to the WM alpha increase. All these regions showed a significant negative correlation with orthogonalized TRL $\alpha$, but not with orthogonalized AVG $\alpha$. Although all these regions showed a significant modulation of BOLD by WM load, the ROI analysis shows that the pattern of activity across the four loads is not in line with the alpha increase. These regions were therefore more likely related to spontaneous non-WMrelated fluctuations in alpha power.

The results detailed above are in line with the inhibition hypothesis relating increased alpha power to top-down functional inhibition of regions that can possibly perturb WM maintenance (Jokisch and Jensen, 2007; Klimesch et al., 2007). The negative correlations between alpha power and reaction time suggest a behavioral relevance of the deactivation of primary visual cortex and of the posterior part of the right middle temporal gyrus further supporting this hypothesis. The analysis of the different alpha subbands revealed that the power increases with load mainly in the upper alpha band, which is in line with observations by Klimesch et al. (1999) in a similar WM task. Decreases in power in the upper alpha band have been related to increased performance in declarative memory tasks (Klimesch 1999).

\section{Frontal theta}

Both inside and outside the MR scanner a significant parametric increase in theta power was detected for the Fz electrode. This midline frontal distribution is typical for the frontal theta response (Asada et al., 1999; Gevins et al., 1997; Onton et al., 2005). Applying ICA not only increased the relative power change, but also increased the timefrequency range for which a significant parametric increase with load was observed. The results also show that the theta response is particularly strong in Load 7.

Based on resting state correlations (Scheeringa et al., 2008) and similar findings by Meltzer et al. (2007) who correlated EEG and fMRI measured in separate sessions, we hypothesized that also during task conditions frontal theta activity would correlate negatively with the BOLD signal in the default mode network (Gusnard and Raichle, 2001; Raichle et al., 2001; Shulman et al., 1997). The results obtained here confirm this. We found that the medial prefrontal cortex, anterior cingulate cortex/precuneus and left and right the angular gyrus correlated negatively with single trial estimates of frontal theta power (TRL $\theta$ ). If we split this regressor in three sources of variance, we saw that these regions also correlate negatively with trial-by-trial fluctuations in frontal theta power (orthogonalized TRL $\theta$ ). In addition, for three of the four regions the pattern of decreased activation with increasing WM load is in line with the observed frontal theta increase. The decrease of the BOLD signal in the right angular gyrus for the higher loads was not significant here, but this region typically is also part of the DMN.

The left and right inferior frontal gyrus showed significant positive single trial correlations modeled in Model I by TRL $\theta$. For model II, no significant effect of WM load was however observed for these regions when analyzed in the conventional way, and no significant relation with orthogonalized AVG $\theta$ was observed. There is only a significant positive relation with trial-by-trial variation modeled by orthogonalized TRL $\theta$. These regions are therefore probably only related to nontask-related fluctuations in frontal theta power.

The question arises whether one or more of the regions in which the BOLD signal correlated with frontal theta is the location where the frontal theta rhythm is generated. Equivalent dipole models report sources of frontal theta to be predominantly in or near the anterior cingulate (Asada et al., 1999; Gevins et al., 1997; Onton et al., 2005; Scheeringa et al., 2008). This is just outside the largest negatively correlating cluster we observed in the medial prefrontal cortex. This discrepancy may be explained by the fact that equivalent dipole models tend to estimate the source location too deep when there is in reality a more superficial distributed source (De Munck et al., 1988). Interestingly, Ishii et al. (1999), who applied a beamformer approach on MEG data, and Miwakeichi et al. (2004) and Martinez-Montes et al. (2004), who applied distributed source models on EEG data, located frontal theta activity in a region in the medial prefrontal cortex that overlaps considerably with the medial frontal region observed here. We therefore assume that the medial frontal cluster is the likely source location for the frontal theta component.

The data presented above strongly suggests that increased frontal theta EEG power can be regarded as a direct consequence of the decreased default mode network activity as measured by fMRI. This notion unites the large body of literature on both topics. Both decreased DMN activity and increased frontal theta activity have been linked to increased cognitive demands. Increased frontal theta is reported in a wide variety of tasks, such as mental arithmetic (Inanaga, 1998; Inouye et al., 1994; Ishihara and Yoshii, 1972; Ishii et al., 1999; Lazarev, 1998; Mizuki et al., 1980; Sasaki et al., 1996; Smith et al., 1999), error detection (Luu et al., 2003; Luu et al., 2004), language 
A

Correlations theta Model I

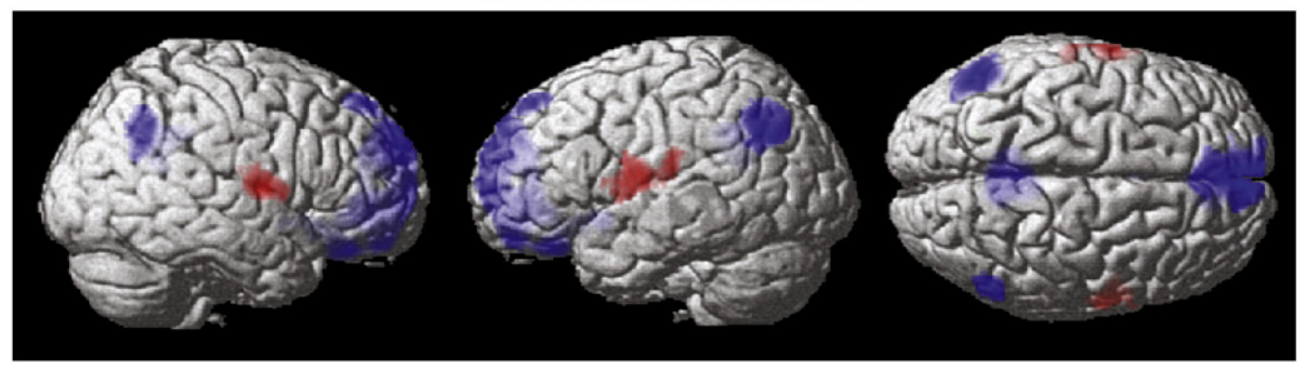

B

Regions related to WM theta increase

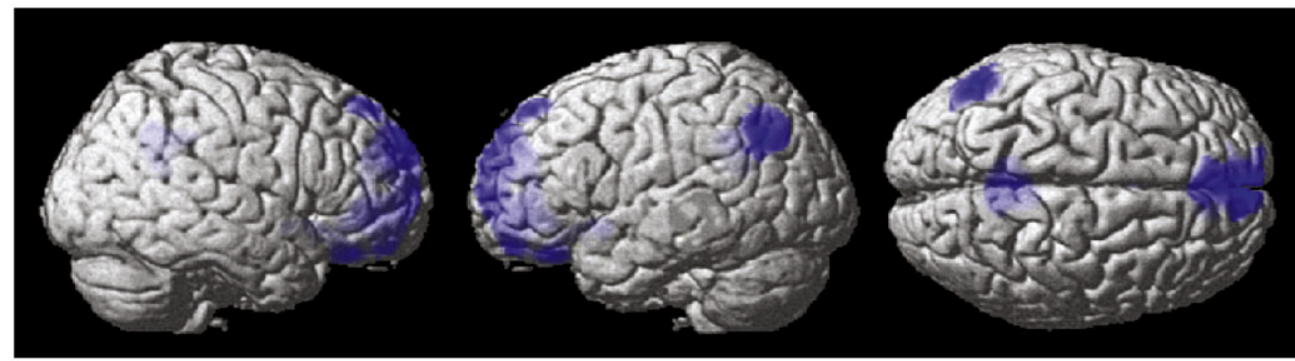

Positive correlations/activations

Negative correlations/deactivations

C
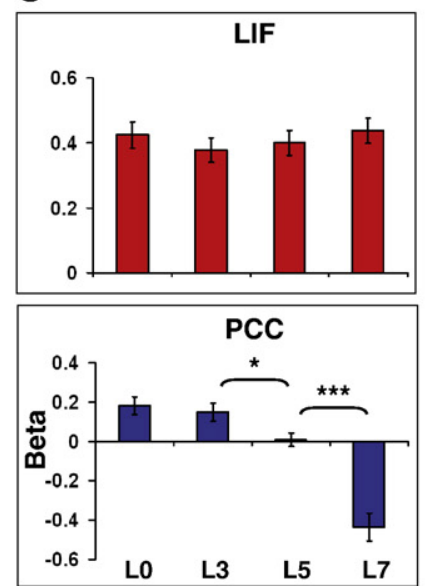

D

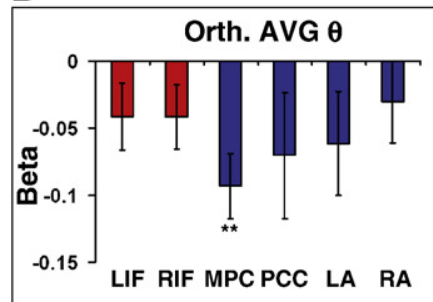

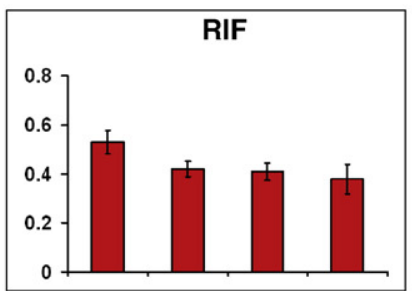

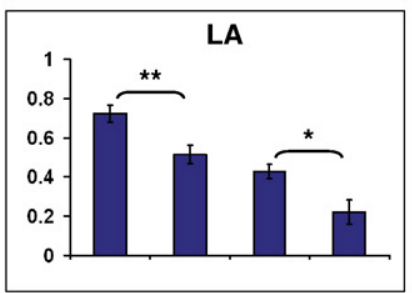

$\mathbf{E}$

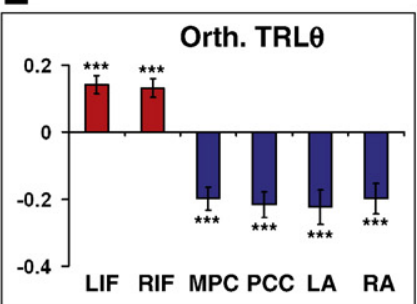

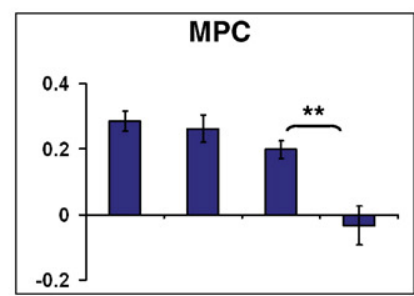

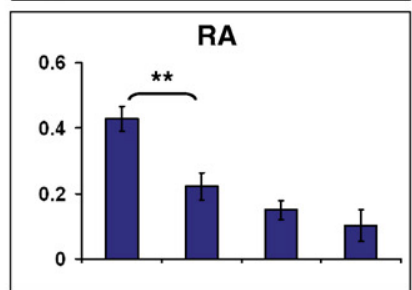

$\mathbf{F}$

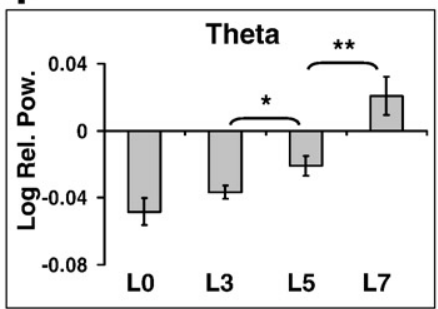

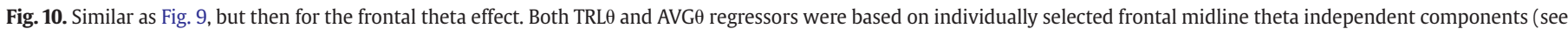

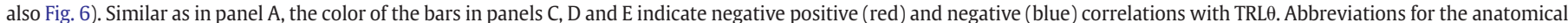

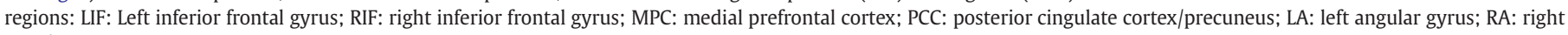
angular gyrus.

comprehension (Bastiaansen et al., 2002; Hald et al., 2006) and WM tasks (Gevins et al., 1997; Jensen and Tesche, 2002; Krause et al., 2000; Onton et al., 2005). DMN network activity as measured by PET and fMRI is reported to decrease in a similarly wide variety of cognitive tasks (Mazoyer et al., 2001; Shulman et al., 1997). Moreover, increased DMN activity has been positively related to activities such as self referential processing (Gusnard et al., 2001) and mind-wandering (Mason et al., 2007). These are activities subjects are typically not engaged in during cognitively demanding tasks. This link between frontal theta activity and DMN activity opens avenues to study activity of default network activity with EEG at sub second level by computing the time course of frontal theta power. 


\section{General issues}

The relation between frontal theta and DMN activity observed here illustrates that EEG power fluctuations measured at scalp level can be related to the activity in larger networks of brain regions and not only to the region that is the direct source of the EEG activity. Not all activity in a functional network is readily detectable with EEG. This study illustrates that concurrent registration of EEG and fMRI can be used to reveal an entire network of regions that is related to changes in EEG features like frequency band specific power modulations. In addition, this study illustrates that by correlating single trial EEG measures, in this case power, with the BOLD signal we can separate different networks that show the same task induced BOLD responses. All the regions that were found to be functionally related to WMrelated increases in either right posterior alpha or frontal theta power show a decrease in BOLD with WM load. By correlating BOLD with single trial alpha and theta power we are able to separate these regions in two separate networks.

We observed here an inverse relationship between the BOLD signal and both right posterior alpha and frontal theta activity. These results are in line with the ideas put forward by Kilner et al. (2005), who concluded on theoretical grounds that increased neural activity should lead to decrease in low frequency power (e.g. delta and theta) and an increase in high frequency power (e.g. beta and gamma). According to their heuristic, the BOLD signal is inversely related to the degree of low versus high frequency components in the EEG. The studies investigating the correlation between electrophysiological measures and hemodynamic measures are largely in line with this hypothesis. Simultaneous recordings of EEG and fMRI have predominantly reported negative correlations with theta (Mizuhara et al., 2004; Scheeringa et al., 2008, present study, but see also: Sammer et al., 2007) and alpha power (Feige et al., 2005; Goldman et al., 2002; Goncalves et al., 2006; Laufs et al., 2006; Laufs et al., 2003a; Moosmann et al., 2003) and positive correlations of the default mode network with beta power in (Laufs et al., 2003b). In anaesthetized cats Niessing et al. (2005) recorded local field potentials while blood oxygenation was simultaneously measured with optical imaging. They reported negative correlations between the two measures in the delta and theta bands and positive correlations in beta and gamma bands during visual stimulation. Especially for the higher gamma frequencies they observed a strong positive correlation with BOLD. This positive BOLD-gamma relation was also observed by Logothetis et al. (2001) who found that the BOLD signal showed a strong positive correlation with gamma band activity observed in local fields potentials (LFP) in monkeys, and Shmuel et al. (2006), who reported that negative BOLD responses go together with a decrease in gamma band activity in the LFP. The majority of studies relating electrophysiological brain signals to hemodynamics thus support the notion of a systematic relation between frequency and direction of the BOLD response, in the sense that low-frequencies are associated with BOLD decreases, while high frequencies are associated with BOLD increases.

Initially, we set out to determine whether the observed increases in alpha and theta power during WM maintenance are related to the regions that are thought to be involved in WM maintenance (mainly dorsolateral prefrontal cortex, DLPFC). We found that alpha power correlated negatively with BOLD in visual cortex and the posterior part of the right temporal gryus, which we link to the inhibition of activity that may disturb the WM maintenance process. Frontal theta power correlated negatively with BOLD in the DMN, suggesting that the presence of theta reflects 'task-orientedness'. Strikingly, both these processes (inhibiting irrelevant information and being engaged in the task) are necessary for the adequate performance of a WM task, but are not central to WM maintenance per se. In other words, what we are missing in our present results is EEG-BOLD correlations in the WM network itself. This begs the question of why we did not observe any correlations between oscillatory EEG activity (recall that we studied a full $1-100 \mathrm{~Hz}$ range) and BOLD signal changes in the usual WM regions (i.e., DLPFC). We see at least three possible answers to this question. First, it may be that DLPFC synchronizes at relatively high (i.e. gamma) frequencies, and that the relatively low amplitudes of gamma oscillations were not reliably detected within the noisy environment of the MR scanner. Second, it may be that WM-related neuronal activity in DLPFC does not show massive synchronization changes, and as a result we did not find any systematic EEG power changes that stem from DLPFC. The third possibility is that the effects were present in other independent components that were not used to construct regressors. Our selection of the components was based on the effects observed in channel level data. An effect that is obscured at channel level by noise or confounds, might be recovered by ICA. Identifying these possible components however is problematic, since we do not have the channel level information in terms of frequency and topography of the effect.

Despite these unresolved issues, the present results illustrate that correlating BOLD signal changes with EEG power fluctuations measured at the scalp while a subject is engaged in a cognitive task yields interpretable and meaningful patterns of activity, which reveal the existence of neuronal activity in functionally coherent, yet spatially distributed networks in the brain. Not all activity in a functional network is readily detectable with scalp EEG. However, our results show that the current approach of a concurrent registration of EEG and fMRI, combined with the appropriate analytic techniques, can uncover both the full spatial extent of functional networks, even if more than one network is simultaneously active during the execution of a given task.

\section{Acknowledgments}

This work was supported by a grant from the Netherlands Organisation for Scientific Research (NWO, grant number 400-03277). We would like to thank Paul Gaalman and Erik van den Boogert for technical assistance and Tom Eichele and Ole Jensen for helpful comments on an earlier version of the manuscript.

\section{References}

Allen, P.J., Josephs, O., Turner, R., 2000. A method for removing imaging artifact from continuous EEG recorded during functional MRI. Neuroimage 12, 230-239.

Allen, P.J., Polizzi, G., Krakow, K., Fish, D.R., Lemieux, L., 1998. Identification of EEG events in the MR scanner: the problem of pulse artifact and a method for its subtraction. Neuroimage 8, 229-239.

Asada, H., Fukuda, Y., Tsunoda, S., Yamaguchi, M., Tonoike, M., 1999. Frontal midline theta rhythms reflect alternative activation of prefrontal cortex and anterior cingulate cortex in humans. Neurosci. Lett. 274, 29-32.

Bastiaansen, M.C.M., van Berkum, J.J.A., Hagoort, P., 2002. Syntactic processing modulates the theta rhythm of the human EEG. Neuroimage 17, 1479-1492.

Bell, A., Sejnowski, T., 1995. An information-maximization approach to blind separation and blind deconvolution. Neural. Comput. 7, 1129-1159.

Benjamini, Y., Hochberg, Y., 1995. Controlling the false discovery rate - a practical and powerful approach to multiple testing. J. R. Stat. Soc. Ser. B. Meth. 57, 289-300.

Cabeza, R., Dolcos, F., Graham, R., Nyberg, L., 2002. Similarities and differences in the neural correlates of episodic memory retrieval and working memory. Neuroimage $16,317-330$.

Cabeza, R., Nyberg, L., 2000. Imaging cognition II: An empirical review of 275 PET and fMRI studies. J. Cogn. Neurosci. 12, 1-47.

D'Esposito, M., Postle, B.R., Rypma, B., 2000. Prefrontal cortical contributions to working memory: evidence from event-related fMRI studies. Exp. Brain Res. 133, 3-11.

De Munck, J.C., Van Dijk, B.W., Spekreijse, H., 1988. Mathematical dipoles are adequate to describe realistic generators of human brain activity. IEEE Trans. Biomed. Eng. 35, 960-966.

Debener, S., Ullsperger, M., Siegel, M., Fiehler, K., von Cramon, D.Y., Engel, A.K., 2005. Trial-by-trial coupling of concurrent electroencephalogram and functional magnetic resonance imaging identifies the dynamics of performance monitoring. J. Neurosci. 25, 11730-11737.

Delorme, A., Makeig, S., 2004. EEGLAB: an open source toolbox for analysis of singletrial EEG dynamics including independent component analysis. J. Neurosci. Methods 134, 9-21.

Eichele, T., Specht, K., Moosmann, M., Jongsma, M.L.A., Quiroga, R.Q., Nordby, H., Hugdahl, K., 2005. Assessing the spatiotemporal evolution of neuronal activation 
with single-trial event-related potentials and functional MRI. Proc. Natl. Acad. Sci. U. S. A. $102,17798-17803$.

Feige, B., Scheffler, K., Esposito, F., Di Salle, F., Hennig, J., Seifritz, E., 2005. Cortical and subcortical correlates of electroencephalographic alpha rhythm modulation. J. Neurophysiol. 93, 2864-2872.

Fletcher, P.C., Henson, R.N., 2001. Frontal lobes and human memory: insights from functional neuroimaging. Brain 124, 849-881.

Fox, M.D., Snyder, A.Z., Zacks, J.M., Raichle, M.E., 2006. Coherent spontaneous activity accounts for trial-to-trial variability in human evoked brain responses. Nat. Neurosci. 9, 23-25.

Gevins, A., Smith, M.E., McEvoy, L., Yu, D., 1997. High-resolution EEG mapping of cortical activation related to working memory: effects of task difficulty, type of processing, and practice. Cereb. Cortex 7, 374-385.

Goldman, R.I., Stern, J.M., Engel, J., Cohen, M.S., 2002. Simultaneous EEG and fMRI of the alpha rhythm. Neuroreport 13, 2487-2492.

Goncalves, S.I., de Munck, J.C., Pouwels, P.J.W., Schoonhoven, R., Kuijer, J.P.A., Maurits, N. M., Hoogduin, J.M., Van Someren, E.J.W., Heethaar, R.M., da Silva, F.H.L., 2006. Correlating the alpha rhythm to BOLD using simultaneous EEG/fMRI: inter-subject variability. Neuroimage 30, 203-213.

Gratton, G., Coles, M.G.H., Donchin, E., 1983. A new method for off-line removal of ocular artifact. Electroencephalogr. Clin. Neurophysiol. 55, 468-484.

Gusnard, D.A., Akbudak, E., Shulman, G.L., Raichle, M.E., 2001. Medial prefrontal cortex and self-referential mental activity: relation to a default mode of brain function. Proc. Natl. Acad. Sci. U. S. A. 98, 4259-4264.

Gusnard, D.A., Raichle, M.E., 2001. Searching for a baseline: functional imaging and the resting human brain. Nat. Rev. Neurosci. 2, 685-694.

Hald, L.A., Bastiaansen, M.C.M., Hagoort, P., 2006. EEG theta and gamma responses to semantic violations in online sentence processing. Brain Lang. 96, 90-105.

Inanaga, K., 1998. Frontal midline theta rhythm and mental activity. Psychiatry. Clin. Neurosci. 52, 555-566.

Inouye, T., Shinosaki, K., Iyama, A., Matsumoto, Y., Toi, S., Ishihara, T., 1994. Potential flow of frontal midline theta-activity during a mental task in the human electroencephalogram. Neurosci. Lett. 169, 145-148.

Ishihara, T., Yoshii, N., 1972. Multivariate analytic study of EEG and mental activity in juvenile delinquents. Electroencephalogr. Clin. Neurophysiol. 33, 71-80.

Ishii, R., Shinosaki, K., Ukai, S., Inouye, T., Ishihara, T., Yoshimine, T., Hirabuki, N., Asada, H., Kihara, T., Robinson, S.E., Takeda, M., 1999. Medial prefrontal cortex generates frontal midline theta rhythm. Neuroreport 10, 675-679.

Jensen, O., 2006. Maintenance of multiple working memory items by temporal segmentation. Neuroscience 139, 237-249.

Jensen, O., Gelfand, J., Kounios, J., Lisman, J.E., 2002. Oscillations in the alpha band (9$12 \mathrm{~Hz}$ ) increase with memory load during retention in a short-term memory task. Cereb. Cortex 12, 877-882.

Jensen, O., Lisman, J.E., 1998. An oscillatory short-term memory buffer model can account for data on the Sternberg task. J. Neurosci. 18, 10688-10699.

Jensen, O., Tesche, C.D., 2002. Frontal theta activity in humans increases with memory load in a working memory task. Eur. J. Neurosci. 15, 1395-1399.

Jokisch, D., Jensen, O., 2007. Modulation of gamma and alpha activity during a working memory task engaging the dorsal or ventral stream. J. Neurosci. 27, 3244-3251.

Kaiser, J., Ripper, B., Birbaumer, N., Lutzenberger, W., 2003. Dynamics of gamma-band activity in human magnetoencephalogram during auditory pattern working memory. Neuroimage 20, 816-827.

Kilner, J.M., Mattout, J., Henson, R., Friston, K.J., 2005. Hemodynamic correlates of EEG: a heuristic. Neuroimage 28, 280-286.

Klimesch, W., 1999. EEG alpha and theta oscillations reflect cognitive and memory performance: a review and analysis. Brain Res. Brain Res. Rev. 29, 169-195.

Klimesch, W., Doppelmayr, M., Schwaiger, J., Auinger, P., Winkler, T., 1999. 'Paradoxical' alpha synchronization in a memory task. Brain Res. Cogn. Brain Res. 7, 493-501.

Klimesch, W., Sauseng, P., Hanslmayr, S., 2007. EEG alpha oscillations: the inhibitiontiming hypothesis. Brain Res. Rev. 53, 63-88.

Krause, C.M., Sillanmaki, L., Koivisto, M., Saarela, C., Haggqvist, A., Laine, M., Hamalainen, H., 2000. The effects of memory load on event-related EEG desynchronization and synchronization. Clin. Neurophysiol. 111, 2071-2078.

Laufs, H., Holt, J.L., Elfont, R., Krams, M., Paul, J.S., Krakow, K., Kleinschmidt, A., 2006 Where the BOLD signal goes when alpha EEG leaves. Neuroimage 31, 1408-1418.

Laufs, H., Kleinschmidt, A., Beyerle, A., Eger, E., Salek-Haddadi, A., Preibisch, C., Krakow, K., 2003a. EEG-correlated fMRI of human alpha activity. Neuroimage 19, 1463-1476.

Laufs, H., Krakow, K., Sterzer, P., Eger, E., Beyerle, A., Salek-Haddadi, A., Kleinschmidt, A., 2003b. Electroencephalographic signatures of attentional and cognitive default modes in spontaneous brain activity fluctuations at rest. Proc. Natl. Acad. Sci. U. S. A. $100,11053-11058$

Lazarev, V.V., 1998. On the intercorrelation of some frequency and amplitude parameters of the human EEG and its functional significance. Communication I: Multidimensional neurodynamic organization of functional states of the brain during intellectual, perceptive and motor activity in normal subjects. Int. J. Psychophysiol. 28, 77-98.

Logothetis, N.K., Pauls, J., Augath, M., Trinath, T., Oeltermann, A., 2001. Neurophysiological investigation of the basis of the fMRI signal. Nature 412, 150-157.

Lutzenberger, W., Ripper, B., Busse, L., Birbaumer, N., Kaiser, J., 2002. Dynamics of gamma-band activity during an audiospatial working memory task in humans. J. Neurosci. 22, 5630-5638.
Luu, P., Tucker, D.M., Derryberry, D., Reed, M., Poulsen, C., 2003. Electrophysiological responses to errors and feedback in the process of action regulation. Psychol. Sci. 14, 47-53.

Luu, P., Tucker, D.M., Makeig, S., 2004. Frontal midline theta and the error-related negativity: neurophysiological mechanisms of action regulation. Clin. Neurophysiol. 115, 1821-1835.

Makeig, S., Westerfield, M., Jung, T.P., Enghoff, S., Townsend, J., Courchesne, E., Sejnowski, T.J., 2002. Dynamic brain sources of visual evoked responses. Science 295, 690-694.

Maris, E., Oostenveld, R., 2007. Nonparametric statistical testing of EEG- and MEG-data. J. Neurosci. Methods 164, 177-190.

Martinez-Montes, E., Valdes-Sosa, P.A., Miwakeichi, F., Goldman, R.I., Cohen, M.S., 2004 Concurrent EEG/fMRI analysis by multiway partial least squares. Neuroimage 22, 1023-1034.

Mason, M.F., Norton, M.I., Van Horn, J.D., Wegner, D.M., Grafton, S.T., Macrae, C.N., 2007. Wandering minds: the default network and stimulus-independent thought. Science 315, 393-395.

Mazoyer, B., Zago, L., Mellet, E., Bricogne, S., Etard, O., Houde, O., Crivello, F., Joliot, M. Petit, L., Tzourio-Mazoyer, N., 2001. Cortical networks for working memory and executive functions sustain the conscious resting state in man. Brain. Res. Bull. 54, 287-298.

Meltzer, J.A., Negishi, M., Mayes, L.C., Constable, R.T., 2007. Individual differences in EEG theta and alpha dynamics during working memory correlate with fMRI responses across subjects. Clin. Neurophysiol. 118, 2419-2436.

Mitra, P.P., Pesaran, B., 1999. Analysis of dynamic brain imaging data. Biophys. J. 76, 691-708.

Miwakeichi, F., Martinez-Montes, E., Valdes-Sosa, P.A., Nishiyama, N., Mizuhara, H. Yamaguchi, Y., 2004. Decomposing EEG data into space-time-frequency components using parallel factor analysis. Neuroimage 22, 1035-1045.

Mizuhara, H., Wang, L.Q., Kobayashi, K., Yamaguchi, Y., 2004. A long-range cortical network emerging with theta oscillation in a mental task. Neuroreport 15, 1233-1238.

Mizuki, Y., Tanaka, M., Isozaki, H., Nishijima, H., Inanaga, K., 1980. Periodic appearance of theta rhythm in the frontal midline area during performance of a mental task. Electroencephalogr. Clin. Neurophysiol. 49, 345-351.

Moosmann, M., Ritter, P., Krastel, I., Brink, A., Thees, S., Blankenburg, F., Taskin, B., Obrig H., Villringer, A., 2003. Correlates of alpha rhythm in functional magnetic resonance imaging and near infrared spectroscopy. Neuroimage 20,145-158.

Niessing, J., Ebisch, B., Schmidt, K.E., Niessing, M., Singer, W., Galuske, R.A., 2005 Hemodynamic signals correlate tightly with synchronized gamma oscillations. Science 309, 948-951.

Onton, J., Delorme, A., Makeig, S., 2005. Frontal midline EEG dynamics during working memory. Neuroimage 27, 341-356.

Petersson, K.M., Gisselgard, J., Gretzer, M., Ingvar, M., 2006. Interaction between a verbal working memory network and the medial temporal lobe. Neuroimage 33,1207-1217.

Piekema, C., Kessels, R.P., Mars, R.B., Petersson, K.M., Fernandez, G., 2006. The right hippocampus participates in short-term memory maintenance of object-location associations. Neuroimage 33, 374-382.

Raichle, M.E., MacLeod, A.M., Snyder, A.Z., Powers, W.J., Gusnard, D.A., Shulman, G.L., 2001. A default mode of brain function. Proc. Natl. Acad. Sci. U. S. A. 98, 676-682.

Ranganath, C., Cohen, M.X., Brozinsky, C.J., 2005. Working memory maintenance contributes to long-term memory formation: neural and behavioral evidence. J Cogn. Neurosci. 17, 994-1010.

Ranganath, C., D'Esposito, M., 2001. Medial temporal lobe activity associated with active maintenance of novel information. Neuron 31, 865-873.

Sammer, G., Blecker, C., Gebhardt, H., Bischoff, M., Stark, R., Morgen, K., Vaitl, D., 2007 Relationship between regional hemodynamic activity and simultaneously recorded EEG-theta associated with mental arithmetic-induced workload. Hum. Brain. Mapp. 28, 793-803.

Sasaki, K., Tsujimoto, T., Nishikawa, S., Nishitani, N., Ishihara, T., 1996. Frontal mental theta wave recorded simultaneously with magnetoencephalography and electroencephalography. Neurosci. Res. 26, 79-81.

Schack, B., Klimesch, W., 2002. Frequency characteristics of evoked and oscillatory electroencephalic activity in a human memory scanning task. Neurosci. Lett. 331, 107-110.

Scheeringa, R., Bastiaansen, M.C., Petersson, K.M., Oostenveld, R., Norris, D.G., Hagoort, P., 2008. Frontal theta EEG activity correlates negatively with the default mode network in resting state. Int. J. Psychophysiol. 67, 242-251.

Shmuel, A., Augath, M., Oeltermann, A., Logothetis, N.K., 2006. Negative functional MRI response correlates with decreases in neuronal activity in monkey visual area V1. Nat. Neurosci. 9, 569-577.

Shulman, G.L., Fiez, J.A., Corbetta, M., Buckner, R.L., Miezin, F.M., Raichle, M.E., Petersen, S.E., 1997. Common blood flow changes across visual tasks .2. Decreases in cerebral cortex. J. Cogn. Neurosci. 9, 648-663.

Smith, M.E., McEvoy, L.K., Gevins, A., 1999. Neurophysiological indices of strategy development and skill acquisition. Brain. Res. Cogn. Brain. Res. 7, 389-404.

Tallon-Baudry, C., Bertrand, O., Peronnet, F., Pernier, J., 1998. Induced gamma-band activity during the delay of a visual short-term memory task in humans. J. Neurosci. $18,4244-4254$

Tuladhar, A.M., Huurne, N.T., Schoffelen, J.M., Maris, E., Oostenveld, R., Jensen, O., 2007. Parieto-occipital sources account for the increase in alpha activity with working memory load. Hum. Brain. Mapp. 